\title{
Advanced Imaging and Direct Targeting of the Motor Thalamus and Dentato-Rubro-Thalamic Tract for Tremor: A Systematic Review
}

\author{
Nicholas Gravbrot ${ }^{\mathrm{a}}$ Manojkumar Saranathan ${ }^{\mathrm{b}}$ Nader Pouratian ${ }^{\mathrm{c}}$ \\ Willard S. Kasoffa \\ ${ }^{a}$ Department of Neurosurgery, University of Arizona College of Medicine, Tucson, AZ, USA; ${ }^{b}$ Department of Medical \\ Imaging, University of Arizona College of Medicine, Tucson, AZ, USA; ' Department of Neurosurgery, David Geffen \\ School of Medicine at UCLA, Los Angeles, CA, USA
}

\section{Keywords}

Essential tremor $\cdot$ Vim nucleus · Dentato-rubro-thalamic tract $\cdot$ Advanced imaging $\cdot$ Direct targeting

\begin{abstract}
Direct targeting methods for stereotactic neurosurgery in the treatment of essential tremor have been the subject of active research over the past decade but have not yet been systematically reviewed. We present a clinically oriented topic review based on Preferred Reporting Items for Systematic Reviews and Meta-Analyses Group guidelines. Our focus is studies using advanced magnetic resonance imaging (MRI) techniques (ultrahigh-field structural MRI, diffusionweighted imaging, diffusion-tensor tractography, and functional MRI) for patient specific, in vivo identification of the ventral intermediate nucleus and the dentato-rubro-thalamic tract.

○) 2020 S. Karger AG, Basel
\end{abstract}

\section{Introduction}

Motor Thalamus Background and Anatomy

A heterogenous structure, the thalamus is composed of multiple gray-matter nuclei with different histological features, anatomical connections, and functional roles, primarily as processing nodes in cortical-subcortical networks involved in motor, sensory, and limbic systems; integrative behavioral processing; sleep; wakefulness; and consciousness [1-3].

The principal nuclei involved in motor circuits are the ventral oral posterior nucleus (Vop) and ventral intermediate nucleus (Vim), as labeled in the Hassler [1] nomenclature favored by surgeons, corresponding approximately to the ventral lateral nucleus anterior portion and ventral lateral nucleus posteroventral portion, respectively, in the Morel [4] nomenclature favored in primate research, the neuroimaging community, and many nonsurgical studies. The Vop and Vim are in the ventrolateral tier of thalamic nuclei, located at the inferior edge of the thalamus, just medial to the internal capsule and anterior to the principal sensory nucleus, labeled the ventral caudal nucleus (corresponding to the ventral posterolateral and ventral posteromedial nuclei in Morel [4] classification). The Vim, as histologically defined, measures approximately $4 \mathrm{~mm}$ in the anteroposterior dimension, $4 \mathrm{~mm}$ mediolaterally, and $6 \mathrm{~mm}$ dorsoventrally [5], representing approximately $0.5-2.0 \%$ of the total thalamic volume $[1,3,4,6]$.

These key ventrolateral nuclei, known as the motor thalamus, are the principal processing and relay points karger@karger.com

www.karger.com/sfn

Karger ${ }^{\prime}=$
(C) 2020 S. Karger AG, Basel
Willard S. Kasoff, MD, MPH

Department of Neurosurgery

University of Arizona College of Medicine, 1501 N. Campbell Ave.

PO Box 245070, Tucson, AZ 85724-5070 (USA)

wkasoff@email.arizona.edu 
between ascending brainstem and cerebellar inputs and motor regions of the cerebral cortex. As such, they have long been a structure of pathophysiologic and therapeutic interest in the treatment of movement disorders [7]. Specifically, the Vim is the most commonly targeted thalamic structure for lesioning and deep brain stimulation (DBS) in the treatment of medically refractory essential tremor (ET) as well as a variety of other tremor syndromes, including parkinsonian tremor, dystonic tremor, and tremor related to cerebellar and brainstem lesions.

\section{Traditional Targeting of the Motor Thalamus}

Because the thalamus lacks sufficient intrinsic contrast on current standard clinical magnetic resonance imaging (MRI) to identify the Vim or other subnuclei [8], surgical targeting has traditionally relied on the use of "indirect" methods, that is, the selection of a target by standard relationships to visible structures. These relationships, based on histological atlases or population-based physiologic data, may be fixed in relation to commissural landmarks or scaled to the patient in a standardized fashion [4, 9-12]. Commonly used fixed coordinates are 13$15 \mathrm{~mm}$ lateral to midline, $6 \mathrm{~mm}$ posterior to the midcommissural point, or, alternatively, anterior to the posterior commissure, at the commissural plane. Numerous systems for scaled coordinates exist, clustering between 10 and $12 \mathrm{~mm}$ lateral to the third ventricular wall, $20-25 \%$ of the distance from the posterior commissure to the anterior commissure, again in the commissural plane. Although robust on its own $[13,14]$, indirect targeting is relatively insensitive to individual variation in the size, location, and configuration of thalamic nuclei [15], and thus it has traditionally been thought to require awake physiologic mapping for electrode or lesion placement, although recent experience using indirect targeting without physiologic feedback has also shown success $[13,14$, $16,17]$. Reliable techniques to directly visualize and target the Vim and other thalamic nuclei on a patient-specific basis would therefore be a useful addition to movement disorders surgery, allowing greater precision and efficiency without the need for awake physiologic testing.

\section{Direct and Advanced Targeting of the Motor Thalamus}

In recent years, a wide variety of MRI-based techniques have been used to parcellate the thalamus and directly visualize the Vim. These include the use of ultrahigh-field strength MRI $[18,19]$, novel MRI sequences at standard clinical field strengths [20,21], clustering based on local or global diffusion-tensor properties [22-25], structural connectivity $[26,27]$, or functional connectiv- ity $[28,29]$. A related focus has been the direct identification of the dentato-rubro-thalamic tract (DRTT) either prior to, or just at, its entrance into the thalamus. These two "direct" targeting approaches are the subject of this review. A separate line of work, which we will not review, has sought to improve on traditional histologic atlases by constructing digitized multipatient atlases using combinations of histology and both in vivo and ex vivo imaging $[6,9,11,30-33]$.

This review focuses specifically on the motor thalamus and DRTT; however, some of the techniques described can be applied to parcellate other functional and anatomic regions of the thalamus, including the anterior nuclear group in the treatment of epilepsy and limbic system disorders [34], specific sensory [35-38], and relay nuclei [39-41] and nuclei implicated in multiple sclerosis [42, 43], depression [44], Alzheimer's disease [45], and Parkinson's disease (PD) [46]. If these methods prove reliable, they may provide valuable opportunities to study pathophysiology in vivo by evaluating volumetric (and, possibly, functional) changes in specific nuclei in a variety of neurodegenerative and psychiatric disorders [47].

\section{Definitions and Imaging Techniques Discussed}

We will use the term structural MRI to refer to standard images based on $\mathrm{T} 1$ and $\mathrm{T} 2$ relaxation times or magnetic susceptibility. Clinical strength MRI refers to 1.5 and 3T; ultrahigh-field strength refers to 7T imaging. Diffusion-tensor imaging (DTI) refers to the characterization of thalamic tissue anisotropy using directional gradients, with subsequent processing techniques to group voxels into clusters with similar properties, without reference to extrathalamic regions of interest (ROI). Diffusion-tensor tractography (DTT) is the application of diffusion imaging to trace white-matter tracts based on voxel-to-voxel streamlines of likely pathways of myelinated fibers, thus indicating structural connectivity between gray-matter ROI. There are 2 main methods of tractography in use: deterministic and probabilistic tractography. Deterministic DTT (dDTT), currently the only method available in commercial neurosurgical planning software, simplifies the anisotropy in a given voxel to a single dominant direction. Streamlines that follow adjacent dominant vectors are then identified between user-specified seed and target regions. Deterministic tractography has the advantage of relative conceptual and computational simplicity but is insensitive to crossing, diverging, "kissing," or other complex fiber arrangements within an individual voxel. Probabilistic DTT (pDTT) retains the multidirectional anisotropy information in the source 
Fig. 1. PRISMA flow diagram.

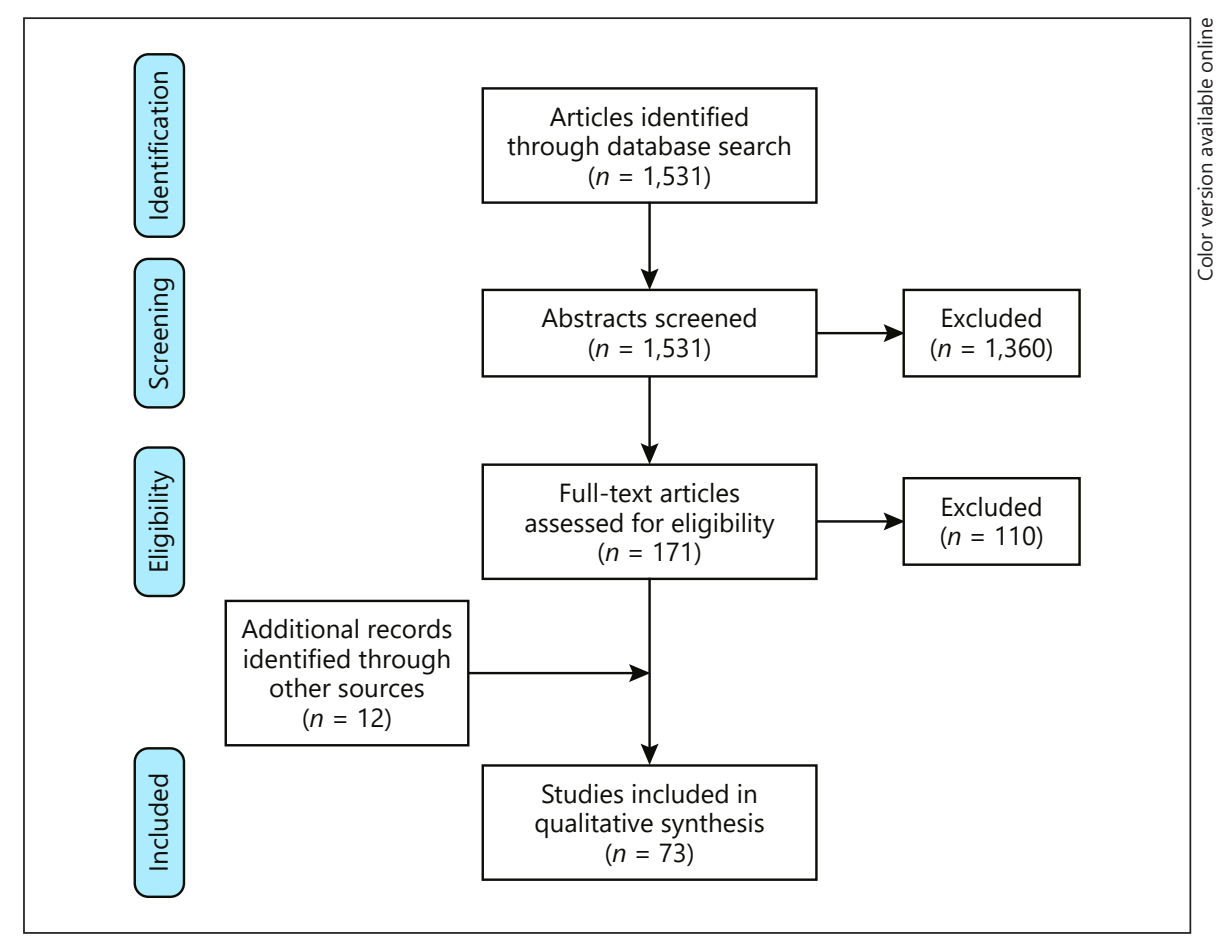

diffusion-weighted imaging data and is thus able to identify, from a given seed area, multiple streamlines, and their relative probability. This method has the advantage of showing more complete information and degree of certainty, as well as greater sensitivity to overlapping fibers and to the pathways of fibers as they exit and enter graymatter structures. However, pDTT is computationally intensive, can be difficult to interpret, and is currently available in research software only. Both dDTT and pDTT are highly dependent on user-defined parameters, such as length and angulation of streamlines and specific ROI selection. Finally, resting-state functional MRI (fMRI, rs-fMRI) identifies individual voxels whose blood oxygen-level-dependent (BOLD) signals vary together over time, suggesting correlation of neuronal activity and thus functional connectivity.

\section{Methods}

A systematic review was conducted according to the Preferred Reporting Items for Systematic Reviews and Meta-Analyses guidelines [48] of articles pertaining to imaging-based thalamic segmentation. Results are outlined in Figure 1. The National Center for Biotechnology Innovation's PubMed database was investigated using the following search strategy: (essential tremor OR Vim OR ventrointermed* OR ventral intermed* OR DRT OR dentatorubrothal* OR dentato-rubro-thal* OR DRTT OR CTCT OR thalamic subnuclei OR thalamic segmentation) AND (direct target
OR parcel* OR segment* OR tractograph* OR diffus* OR connect* OR fMRI). This yielded 1,531 unduplicated results. These results were reviewed by 2 authors (N.G., W.K., with differences resolved by the senior author) using the following inclusion criteria: (a) human studies, (b) in vivo imaging, (c) parcellation of the motor thalamus (Vim, Vop, or ventrolateral motor regions as defined by structural or functional connectivity) or DRTT, and (d) use of 1 of the imaging techniques listed above. Single-patient case reports and studies reporting the creation of new atlases were excluded. Of note, although the DRTT passes through posterior subthalamic area (PSA) and caudal zona incerta (cZI), studies of those 2 white-matter targets using conventional imaging were excluded to preserve a focused topic review. The PSA and cZI targets have recently been thoroughly reviewed [49].

\section{Results}

\section{Structural MRI}

A total of 17 articles were identified for analysis. Of these, 8 reported clinical correlation in surgical patients. The remainder were studies with healthy controls, using atlas comparison for verification. Study characteristics are summarized in Table 1.

Several groups have sought to parcellate the thalamus using intrinsic signal characteristics, primarily exploiting differences in myelin content of subnuclei, nuclear groups, and the laminae separating them. Kawashima et al. [50], using a proton-density sequence at $1.5 \mathrm{~T}$, were 
Table 1. Structural MRI studies

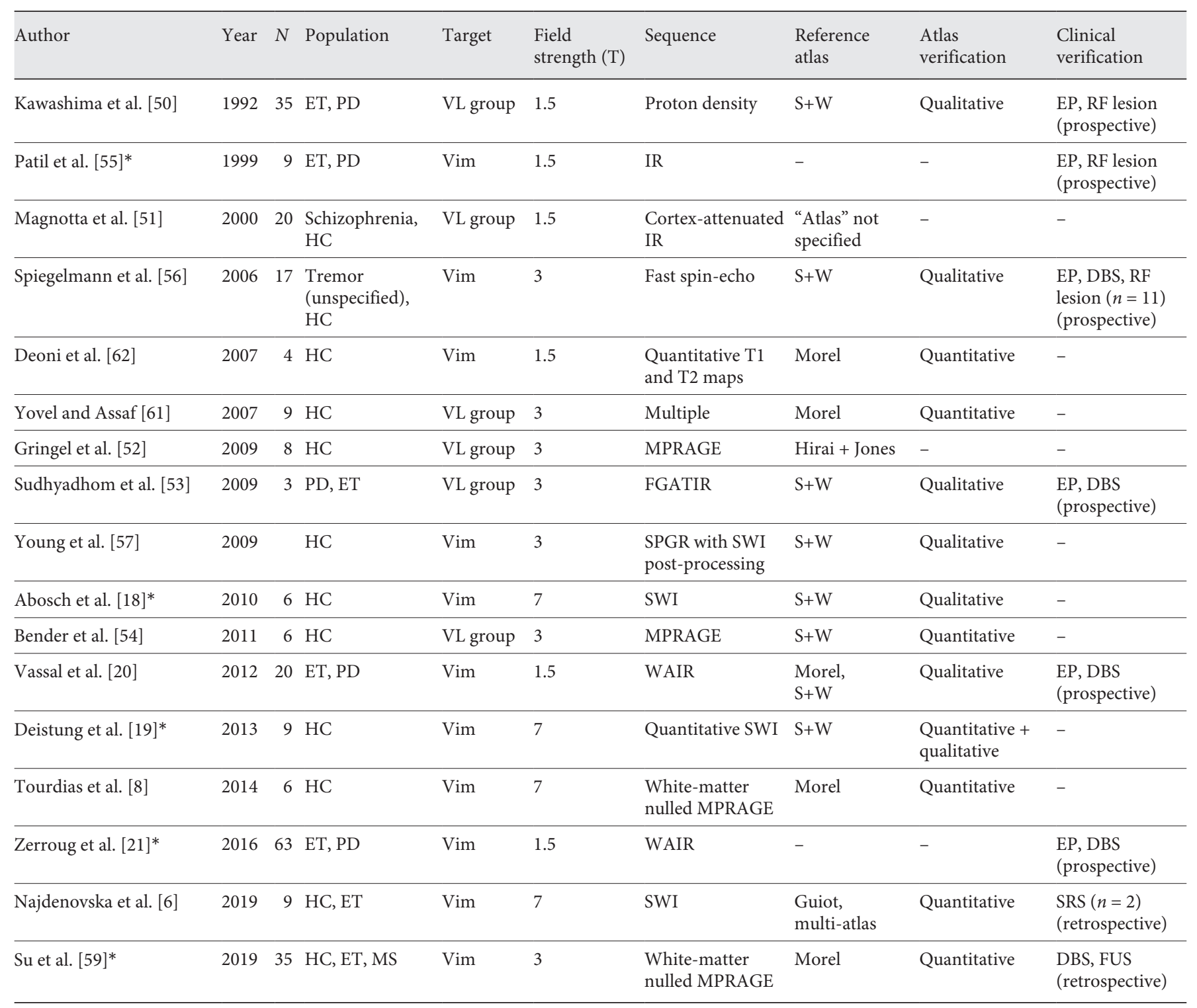

* Study of particular interest or importance. DBS, deep brain stimulation; EP, electrophysiology; ET, essential tremor; FGATIR, fast gray matter acquisition T1 inversion recovery; FUS, focused ultrasound thalamotomy; HC, healthy controls; IR, inversion recovery; MPRAGE, magnetization-prepared rapid acquisition with gradient echo; $\mathrm{N}$, number of participants; PD, Parkinson's disease; RF, radiofrequency; SPGR, spoiled gradient recalled acquisition in steady state; SRS, stereotactic radiosurgery; S+W, Schaltenbrand-Wahren; SWI, susceptibility-weighted imaging; Vim, ventral intermedius nucleus; VL, ventral lateral nucleus; WAIR, white matter attenuated inversion recovery; MRI, magnetic resonance imaging.

able to distinguish the VL nuclear group from the medial, dorsal, and anterior groups, using intraoperative physiology for further refinement. Similar results (at the level of nuclear groups only) were obtained by Magnotta et al. [51] and Gringel et al. [52] at 3T using inversion-recovery sequences and by Sudhyadhom et al. [53] and Bender et al. [54] at 3T using modified magnetization-prepared rapid acquisition with gradient echo sequences.

Direct Targeting of the Motor Thalamus
Further refinement to identify the Vim within the ventrolateral group has also been described at clinical field strength. Patil et al. [55], using an inversion-recovery sequence at $1.5 \mathrm{~T}$, visualized the Vim as a hypointense band extending medially from the internal capsule in the midportion of the thalamus. Using this target for DBS placement and noting significant difference from indirect coordinates, tremor freedom was obtained in all 9 patients 


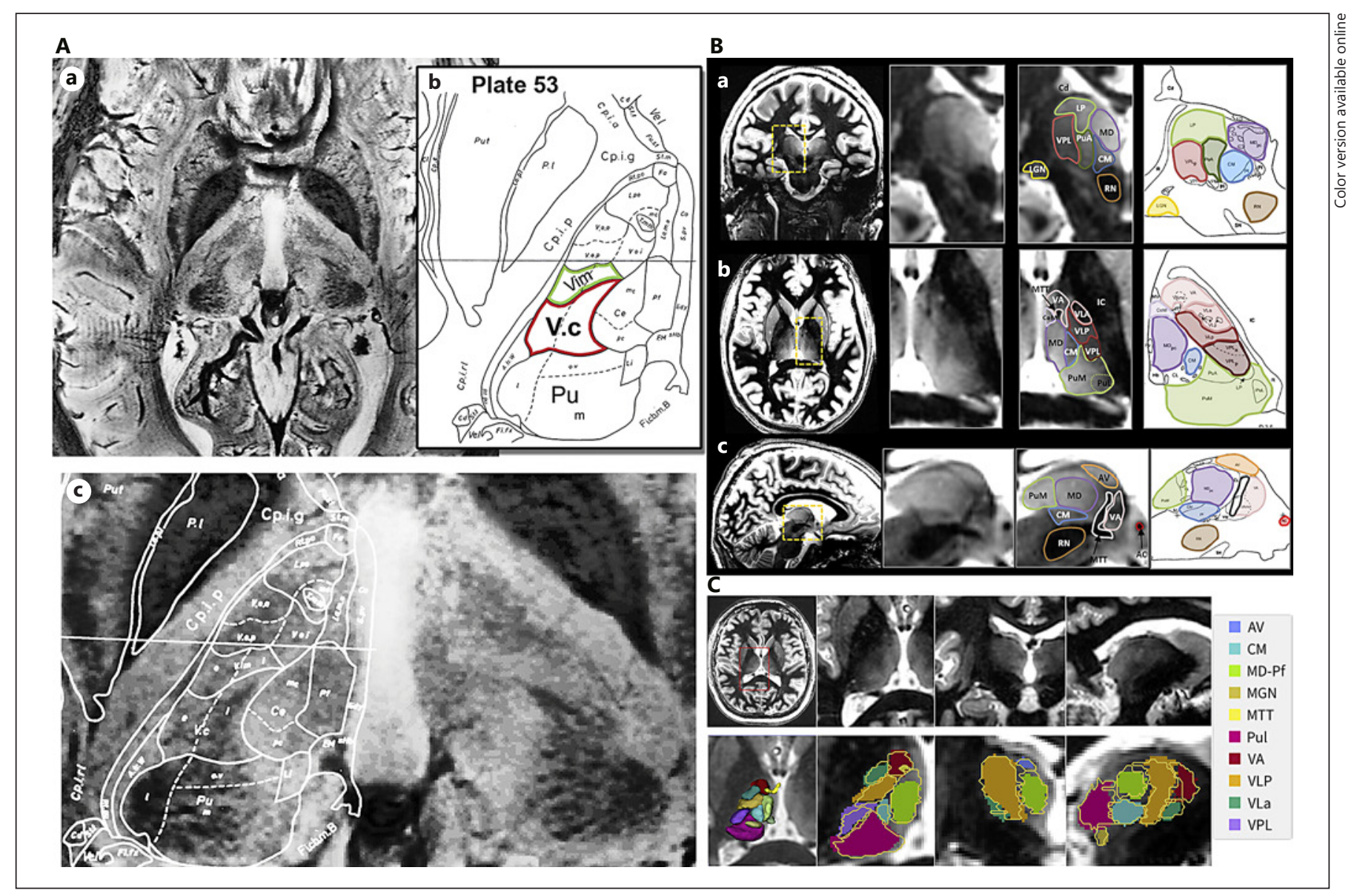

Fig. 2. Ultrahigh-field MRI. A Internal architecture of the thalamus as revealed by SWI at 7T. The Vim and Vc are visible on an axial slice (a), as outlined on Plate 53 from the Schaltenbrand-Wahren atlas (b), and on the combined image (c). Reproduced with permission from Abosch et al. [18]. B White-matter-nulled fast graymatter acquisition T1 inversion recovery imaging at 7T reveals thalamic subnuclei corresponding to the Morel atlas. Reproduced with permission from Tourdias et al. [8]. C A multipatient atlas based on Tourdias et al. [8] is used to automatically segment a $3 \mathrm{~T}$ white-matter-nulled image. Segmented areas correspond well with manually drawn outlines (thin borders). Reproduced with permission from Su et al. [59].

with a single electrode pass. Similar findings of a hypointense, coronally oriented band corresponding to atlasbased location of the Vim were described by Spiegelmann et al. [56] and Young et al. [57]; Young's group did not describe clinical correlation, however. More recently, Vassal et al. [20] and Zerroug et al. [21], using a whitematter-attenuated inversion-recovery sequence at $1.5 \mathrm{~T}$, targeted the same hypointense band during awake DBS placement. Using this target, the DBS lead was implanted in the first trajectory in $84.5 \%$ of patients. It is worth noting, however, that a similar success rate would be expected with standard indirect targeting.

Greater spatial resolution and signal-to-noise ratio have been obtained by several groups using ultrahigh- field strength (7T) MRI, enabling enhanced direct visualization of the Vim. Examples are provided in Figure 2. Abosch et al. [18], using susceptibility-weighted imaging (SWI) in 7 healthy volunteers, showed excellent delineation of the Vim and other nuclear borders, with good correlation to atlas overlays. Similar detail was seen by Deistung et al. [19] using quantitative magnetic susceptibility mapping in 9 healthy volunteers. Tourdias et al. [8] used an optimized white-matter-nulled magnetization-prepared rapid acquisition with gradient echo sequence at $7 \mathrm{~T}$ in 5 healthy controls to manually segment 11 thalamic nuclei, including the Vim. The same group demonstrated clinical correlation of Vim segmentation with FUS lesioning [58]. More recently, the same group's segmenta- 
Table 2. Diffusion-tensor imaging studies

\begin{tabular}{|c|c|c|c|c|c|c|c|c|c|}
\hline Author & Year & $N$ & Population & Target & $\begin{array}{l}\text { Field } \\
\text { strength }(\mathrm{T})\end{array}$ & $\begin{array}{l}\text { Clusters, } \\
n\end{array}$ & $\begin{array}{l}\text { Reference } \\
\text { atlas }\end{array}$ & $\begin{array}{l}\text { Atlas } \\
\text { verification }\end{array}$ & $\begin{array}{l}\text { Clinical } \\
\text { verification }\end{array}$ \\
\hline Wiegell et al. [22] & 2003 & 4 & $\mathrm{HC}$ & VL group & 1.5 & 14 & Niemann & Quantitative & - \\
\hline Ziyan et al. [102] & 2006 & 10 & $\mathrm{HC}$ & $\begin{array}{l}\text { Ventral } \\
\text { group }\end{array}$ & 3 & 12 & $\begin{array}{l}\text { Not } \\
\text { specified }\end{array}$ & Quantitative & - \\
\hline Sedrak et al. $[64]^{*}$ & 2011 & 4 & ET, PD & Vim & 1.5 & $\mathrm{~N} / \mathrm{a}$ & $\mathrm{S}+\mathrm{W}$ & Qualitative & $\begin{array}{l}\text { DBS } \\
\text { (retrospective) }\end{array}$ \\
\hline Mang et al. [23] & 2012 & 63 & $\mathrm{HC}$ & VL group & 1.5 & 21 & Morel & Quantitative & - \\
\hline
\end{tabular}

* Study of particular interest or importance. DBS, deep brain stimulation; ET, essential tremor; HC, healthy controls; $N$, number of participants; PD, Parkinson's disease; S+W, Schaltenbrand-Wahren; Vim, ventral intermedius nucleus; VL, ventral lateral nucleus.

tion was automated using a multiatlas segmentation method by Su et al. [59] and tested on 20 healthy controls and 15 patients with multiple sclerosis, with Dice coefficient of 0.79 for ventral lateral posterior nucleus (compared to manual segmentation guided by Morel atlas). Najdenovska et al. [6] recently reported 9 healthy controls, and 2 ET patients analyzed with 7T SWI imaging based on the work of Abosch et al. [18] In their study, the manually delineated Vim at 7T correlated well with the Vim target chosen by Guiot's method [60] and with automated multiatlas and DTI-based segmentation by their group's previously reported method (see Battistella et al. [25] below). Guiot's point was within or at the edge of the 7T-visible Vim in all cases and in the ventral portion in 7 of 9 cases. The calculated Dice overlap of the 7T-visible Vim with automated multiatlas segmentation was $0.45 \pm$ 0.13 for the left and $0.45 \pm 0.26$ for the right hemisphere.

A small number of publications have attempted to identify the Vim by combining the information from different MRI sequences [61] or by using quantitative MR mapping to "fingerprint" the nucleus [62], but these have been met with limited success and have not been subject to clinical correlation.

In summary, structural MRI at clinical field strengths may show the ventrolateral group and possibly Vim, but reports of clinical correlation or quantitative evaluation against an anatomical atlas have been relatively rare. Newer white-matter-attenuated sequences show potential promise in this regard [59]. 7T MRI provides powerful resolution that appears to allow direct identification of the Vim and Vop; however, prospective application of ultrahigh-field MRI for targeting in individual patients has not yet been reported, and high-field human MRI requires significant financial commitment and institutional expertise, limiting clinical applicability.

\section{Diffusion-Tensor Imaging}

Nine publications were included within this group, 1 with clinical correlation. Table 2 provides a summary of the findings.

In 2003, Wiegell et al. [22] evaluated a semi-automated algorithm to define 14 thalamic clusters based on intrinsic anisotropy using 1.5T diffusion-weighted imaging in 4 healthy controls. The resulting 3-dimensional map was compared with multiple atlases for labeling of nuclei. Major nuclei and some subnuclei (including ventral lateral posterior nucleus) were identified in all $4 \mathrm{sub-}$ jects, although not all with center-of-mass coordinates consistent with prior atlases. The k-means clustering algorithm used in this study has been subject to criticism by later groups, who have used alternative analyses that are more robust to elongated nuclear shapes and more 


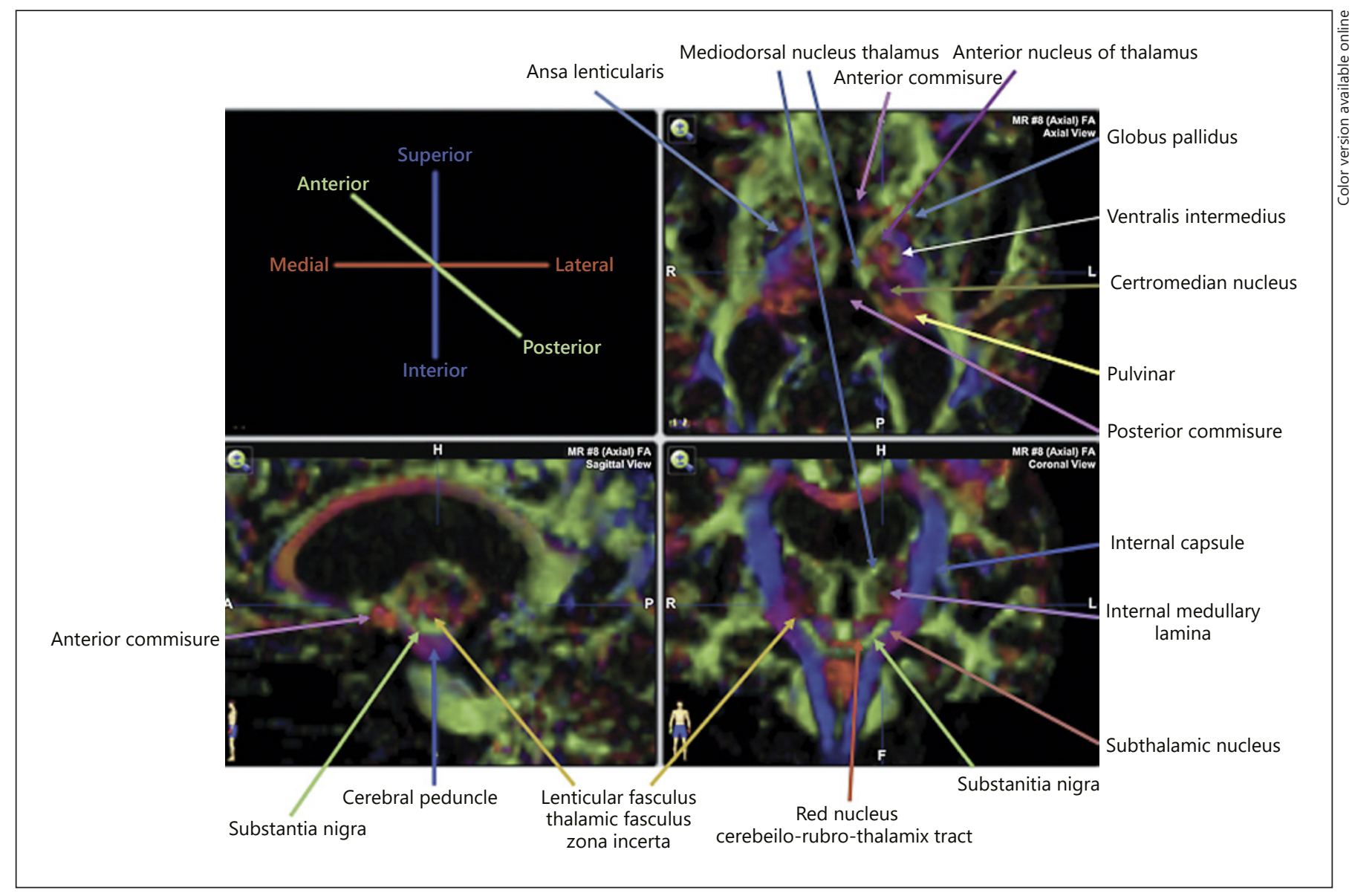

Fig. 3. DTI. Colored fractional anisotropy map at $1.5 \mathrm{~T}$ indicates fiber direction (red, medial - lateral, blue, superior - inferior, green, anterior - posterior). The Vim is seen in the ventrolateral thalamus, superior to multiple crossing fibers, and medial to the corticospinal tracts. Reproduced with permission from Sedrak et al. [64]. FA, fractional anisotropy.

robust to noise. An additional 3 publications from another group $[23,24,63]$ have also used intrinsic diffusion properties to divide the thalamus into 21 clusters based on dominant eigenvector. Individual patient maps were highly variable and difficult to compare to either the Morel atlas or a structural connectivity-based atlas; however, group-level analysis did identify parts of 5 clusters which were common to $90 \%$ of subjects. On inspection, these corresponded roughly with Morel nuclear clusters (although not individual nuclei), and pDTT with seeds in these 5 clusters seemed to reproduce the expected connectivity of those regions, including connection of the ventrolateral cluster to the premotor cortex. More recently, Battistella et al. [25] presented an algorithm for automated parcellation of the thalamus into 7 nuclear groups, including a ventrolateral group, and showed consistent parcellation across all subjects, although no clinical correlation or quantitative comparison to an anatomic atlas was made.

In the only DTI-based study with clinical correlation, Sedrak et al. [64] conducted a retrospective review of 4 Vim DBS patients (3 ET, 1 PD) selected for excellent lead placement (insertional effect, stimulation at $<2.0 \mathrm{~V}$ ). Lead location was found to correlate with characteristic findings on color fractional anisotropy maps, suggesting that the Vim nucleus can be identified based on intrinsic anisotropy (Fig. 3). Deterministic tractography was performed separately using 3 different seeds: the ROI defined by anisotropy, the location of the active DBS contact, and the Vim as determined by the Schaltenbrand-Wahren atlas. All 3 ROIs demonstrated connectivity to the sensorimotor cortex and cerebellum via the superior cerebellar peduncle (SCP), providing validation across methods. Although without clinical validation, O'Muircheartaigh 
Table 3. Probabilistic diffusion-tensor tractography studies

\begin{tabular}{|c|c|c|c|c|c|c|c|c|}
\hline Author & Year & $N$ & Population & Target & ROIs & $\begin{array}{l}\text { Reference } \\
\text { atlas }\end{array}$ & $\begin{array}{l}\text { Atlas } \\
\text { verification }\end{array}$ & $\begin{array}{l}\text { Clinical } \\
\text { verification }\end{array}$ \\
\hline Behrens et al. [26]* & 2003 & 8 & $\mathrm{HC}$ & Vim, Vop & $\begin{array}{l}\text { Thal, M1 or } \\
\text { premotor cortex }\end{array}$ & Morel & Qualitative & - \\
\hline Johansen-Berg et al. [68] & 2005 & 11 & $\mathrm{HC}$ & Vim, Vop & Per Behrens 2003 & Morel & $\begin{array}{l}\text { Quantitative + } \\
\text { qualitative }\end{array}$ & - \\
\hline O’Muircheartaigh et al. [70]* & 2011 & 38 & $\mathrm{HC}$ & Vim, Vop & Per Behrens 2003 & Morel & - & - \\
\hline Pouratian et al. [71]* & 2011 & 6 & ET & Vim, Vop & Per Behrens 2003 & - & - & DBS (retrospective) \\
\hline Hyam et al. [105] & 2012 & 17 & $\mathrm{HC}$ & Vim, Vop & Vim, Vop & - & - & - \\
\hline Klein et al. [73] & 2012 & 12 & $\mathrm{ET}$ & Vim & $\begin{array}{l}\text { Seed on effective } \\
\text { DBS contact }\end{array}$ & $\mathrm{S}+\mathrm{W}$ & Qualitative & DBS (retrospective) \\
\hline Sudhyadhom et al. [12]* & 2013 & 5 & ET & VL group & Per Behrens 2003 & $\mathrm{~S}+\mathrm{W}$ & - & $\mathrm{EP}$ (retrospective) \\
\hline O’Muircheartaigh et al. [29]* & 2015 & 102 & $\mathrm{HC}$ & Vim, Vop & Per Behrens 2003 & - & - & - \\
\hline Jakab et al. [65] & 2016 & 4 & $\mathrm{HC}$ & VLpv & Per Kwon 2011 & Morel & Quantitative & - \\
\hline O’Halloran et al. [98] & 2016 & 2 & $\mathrm{PD}$ & СТCT & $\begin{array}{l}\text { Seed on DBS } \\
\text { contacts }\end{array}$ & - & - & DBS (retrospective) \\
\hline Lambert et al. [66] & 2017 & 40 & $\mathrm{HC}$ & VL group & Thal only & Morel & Quantitative & - \\
\hline
\end{tabular}

* Study of particular interest or importance. CDR, connectivity-defined region; CTCT, cerebello-thalamo-cortical tract; DBS, deep brain stimulation; DN, dentate nucleus; DRTT, dentato-rubro-thalamic tract; EP, electrophysiology; ET, essential tremor; FUS, focused ultrasound thalamotomy; HC, healthy controls; M1, primary motor cortex; N, number of participants; PD, Parkinson's disease; RN, red nucleus; ROI, region of interest; SCP, superior cerebellar peduncle; S+W, Schaltenbrand-Wahren; Thal, thalamus; Vim, ventral intermedius nucleus; VL, ventral lateral nucleus; VLa, ventral lateral nucleus, anterior portion; VLp, ventral lateral nucleus, posterior portion; VLpv, ventral lateral nucleus, posteroventral portion; Vop, ventral oral posterior nucleus.

et al. [29] performed a similar segmentation based on probabilistic tensors, showing that cortical functional connectivity of the resulting 7 thalamic segments corresponded to the known functional circuits with the expected anterior-to-posterior arrangement.

Diffusion-based methods rely on echo-planar imaging, which is intrinsically limited to $2 \mathrm{~mm}$ (typically $2-$ $3 \mathrm{~mm}$ ) isotropic spatial resolution and is also subject to distortion. Furthermore, these methods rely on anisotropic properties of white matter, whereas the thalamus is a largely gray-matter structure, resulting in noisy estimates of the principal tensor orientation. Not surprisingly, the most stable method [25] is confined to 7 nuclei, 1 of which is a composite of anatomic nuclei and does not correspond to a physical nucleus.

\section{Diffusion-Tensor Tractography}

Tractography-aided targeting of the Vim and DRTT has been the subject of extensive investigation over the past 15 years. Techniques generally fall into 2 broad cat- 
egories. The first approach uses probabilistic tractography to group individual thalamic voxels into connectivity-defined regions (CDR) based on their pattern of cortical connectivity, with the CDRs showing preferential connectivity to premotor or motor cortex being classified as Vop or Vim, respectively. The second approach aims to image the axons of the DRTT itself, usually with deterministic tractography seeded at known DRTT waypoints. Within these categories, studies are heterogeneous in their selection of ROIs and exclusion zones, thresholding, and other DTT parameters. Even similar methods using different MRI manufacturers may yield different results $[65,66]$.

Structural Connectivity-Defined Segmentation of the Thalamus Using pDTT

Nineteen probabilistic tractography papers were identified, 8 with clinical correlation (all retrospective). Results are summarized in Table 3.

Behrens et al. [26] pioneered the use of pDTT in parcellating the thalamus and defining its cortical connectivity in vivo. The cerebral cortex was divided into 7 regions, including primary motor and premotor cortex. The probability of connectivity with each cortical region was calculated for each $1.7 \times 1.7 \times 2.3 \mathrm{~mm}$ thalamic voxel, with each voxel ultimately assigned to the single cortical region with which it was most strongly connected (the "winnertake-all" approach). The 7 thalamic CDRs thus identified bore a strong resemblance to histological regions as defined by the Morel atlas, with the CDRs corresponding to primary motor cortex and premotor cortex being found in the expected regions of the ventral lateral and ventral anterior nuclei, respectively (Fig. 4). This foundational work has provided the model and methodology for much of the subsequent use of pDTT in thalamic segmentation, including validation in a pediatric cohort [67]. Subsequent work by the same group [68] demonstrated that the CDRs obtained by the method above colocalized with thalamic divisions on fMRI, providing supporting evidence of a structural - functional overlap. The collected structural connectivity data from 11 normal subjects used in these 2 studies have been placed online as the Oxford Connectivity Atlas.

Kwon et al. [69] used probabilistic tractography with subcortical rather than cortical ROIs, placing seeds for the DRTT in the dentate nucleus and SCP contralateral to, and the red nucleus (RN) ipsilateral to the thalamic side of interest. Although corresponding figures were not shown, the resulting thalamic areas of highest connectivity were reported to be consistent with the expected loca- tion of the ventral lateral nucleus posteroventral portion per the Morel atlas.

O'Muircheartaigh et al. [70] analyzed pDTT data using both the Behrens et al. [26] cortical masks and an independent component analysis (ICA) that was able to assign thalamic voxels in a hypothesis-free manner based on connectivity. The resulting CDRs showed good overlap and reproduction of the Behrens parcellation. The authors argue that ICA provides a more conservative, and therefore reliable, estimate of nuclear location. Their probabilistic assignment of voxels is also likely more physiologically realistic than the "winner-take-all" approach, given the relatively large voxel size of $\sim 2 \mathrm{~mm}^{3}$. Subsequent work by the same group [29] showed that rs-fMRI seeds placed on their CDRs indeed showed functional, as well as structural, connectivity to the expected functional areas of cortex.

Pouratian et al. [71] provided the first illustration of pDTT methods used to analyze a thalamic target for DBS in clinical practice. The pDTT segmentation method of Behrens et al. [26] was applied retrospectively to the preoperative MRI of 10 ET patients who had undergone standard Vim DBS placement using indirect targeting and physiologic mapping. The location of the effective DBS contact on postoperative imaging was found to highly correlate with the supplementary motor area (SMA)/premotor cortex CDR. This finding was consistent across subjects despite interindividual variations in CDR location. The electrode location, at the border between the primary motor and SMA/premotor CDRs, suggested that pDTTbased structural connectivity can be used in individual patients to identify the DBS target at the border between Vop and Vim. Similar results were seen by Middlebrooks et al. [72] in a larger, retrospective series of $40 \mathrm{ET}$ patients who had undergone awake Vim DBS using a locally derived atlas for initial targeting. Strong correlation with tremor reduction was seen for overlap of the volume of tissue activated (VTA) with the SMA/premotor CDR, with no such correlation found with the M1-defined CDR.

Klein et al. [73] also retrospectively evaluated connectivity patterns in 12 patients who had undergone standard awake Vim DBS placement. Effective contact positions, although variable on a scaled Schaltenbrand-Wahren atlas, showed consistent CDR patterns. In contrast to the above studies, however, a stronger connection was found to primary rather than supplementary motor cortex, but significant overlap was seen. Akram et al. [27] also found that DBS VTAs within the M1-defined CDR or the associated cerebello-thalamo-cortical tract (CTCT) were effective, whereas VTAs outside those areas were ineffective (Fig. 4). In 
Fig. 4. Probabilistic diffusion-tensor tractography. A Each thalamic voxel is labeled based on the predefined cortical area (a) with which it has highest degree of structural connectivity. This approach produces subnuclear arrangements that resemble histologic maps (b-d). Reproduced with permission from Behrens et al. [26]. B A later publication by the same group illustrates cortical and cerebellar region-of-interest connectivity to more precisely outline the motor and sensory thalamic nuclei. The overlap of primary motor and dentate connectivity indicates Vim. Reproduced with permission from Akram et al. [27]. SMA/PMC, supplementary motor area/ premotor cortex.
A
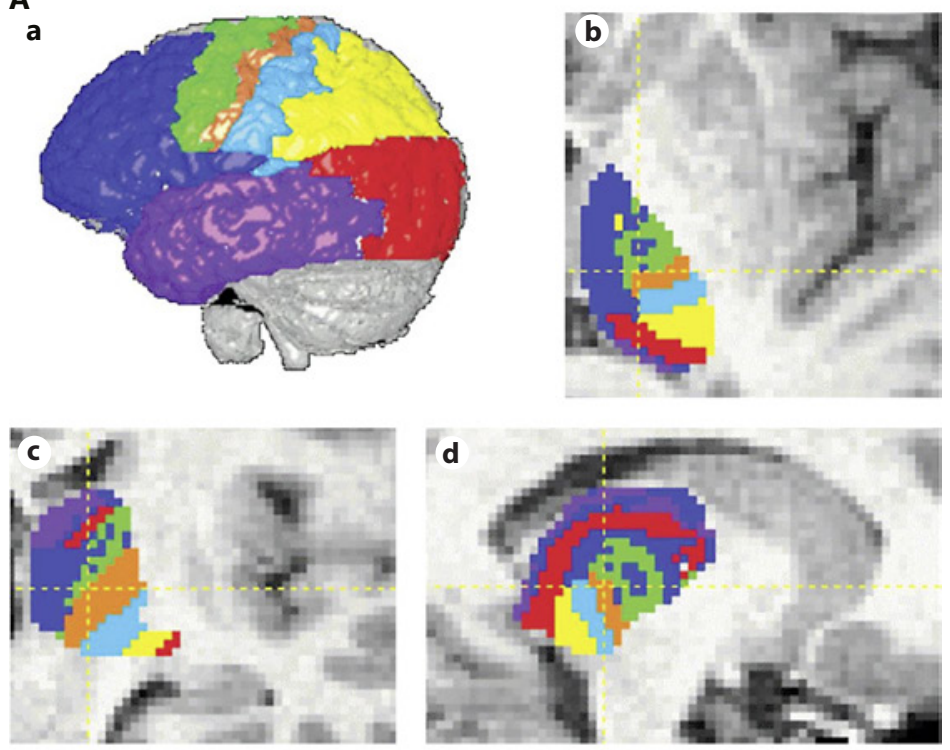

B
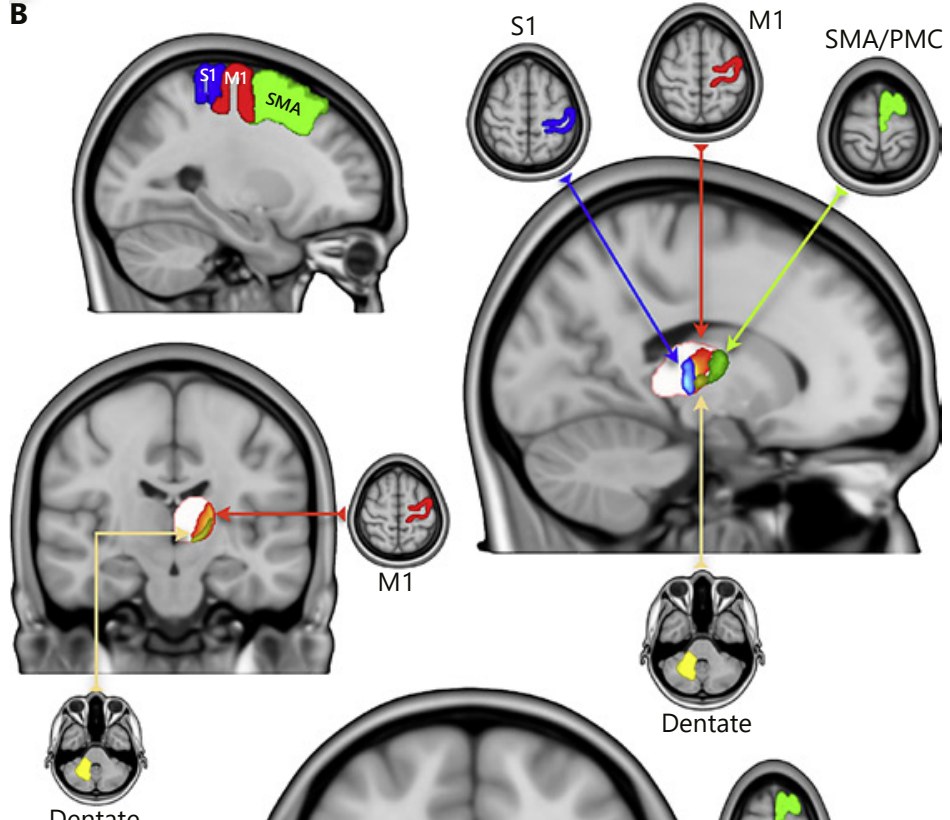

Dentate

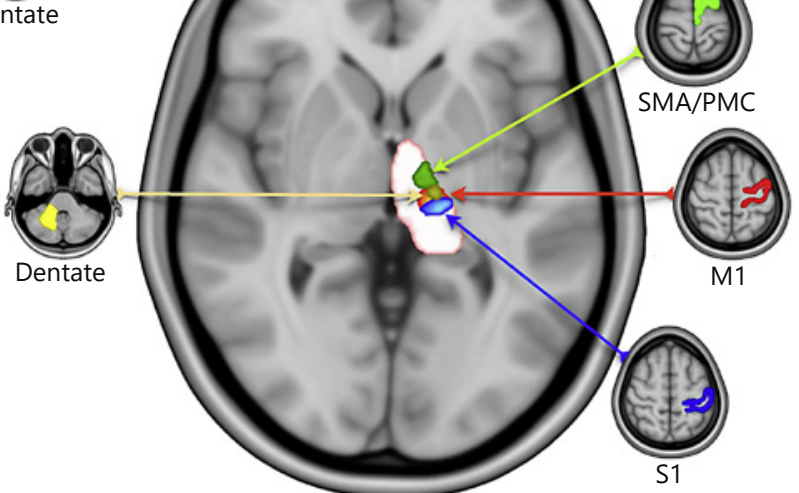

Stereotact Funct Neurosurg 2020;98:220-240 DOI: $10.1159 / 000507030$ 
Table 4. Deterministic diffusion-tensor tractography studies

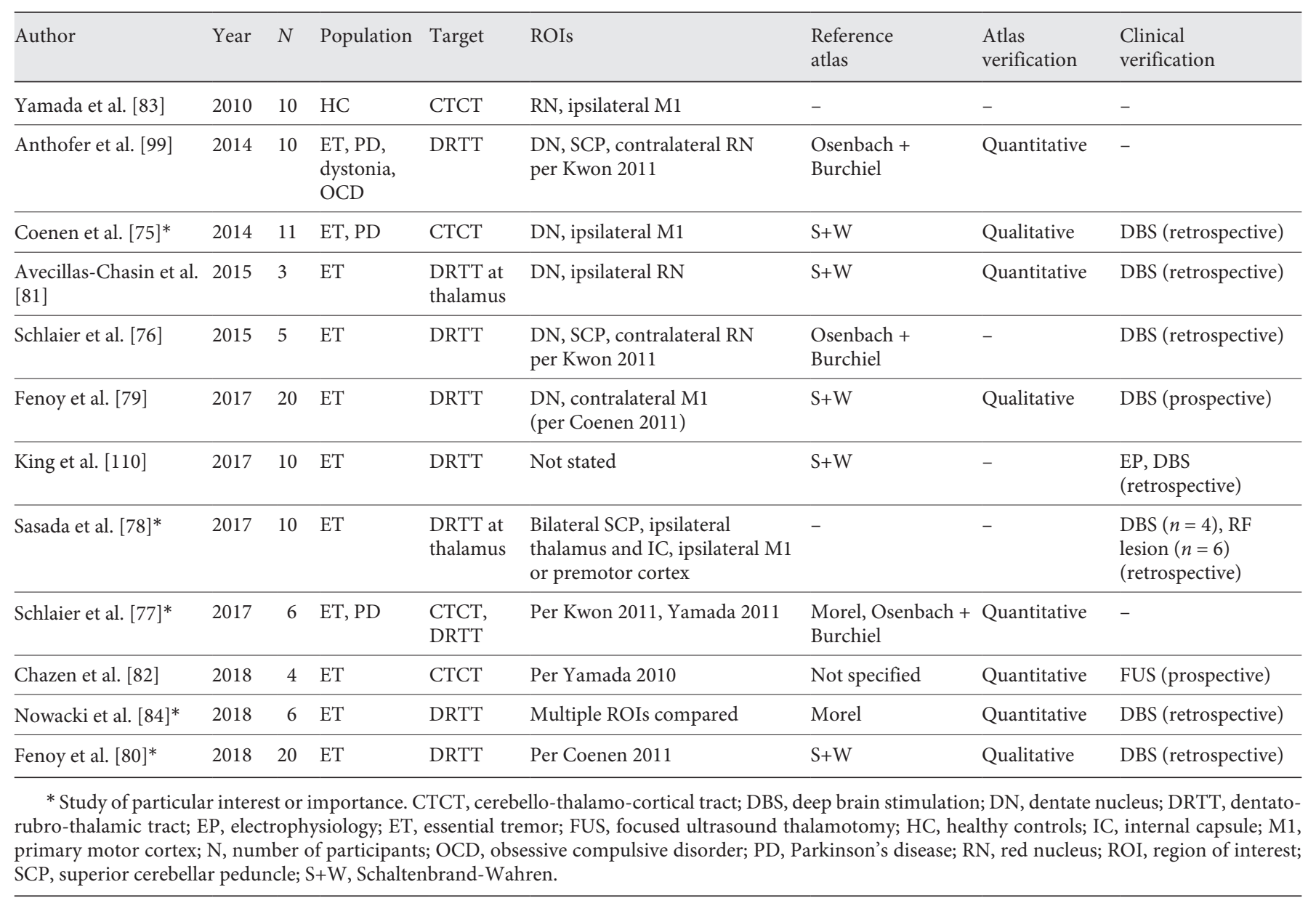

Sudhyadhom et al.'s [12] study of 5 ET patients who underwent awake Vim DBS placement, post hoc analysis showed good agreement of microelectrode data with the somatosensory CDR but only fair agreement with motor CDRs. Allowing mixed assignment of voxels to motor and sensory regions showed better performance, again suggesting that functional areas may overlap structurally or cannot be fully resolved with current voxel sizes. In these 5 patients, in contrast to Klein's findings, microelectrode recording results showed good agreement with a scaled SchaltenbrandWahren atlas, raising the question of whether pDTT-based targeting would represent a clinically meaningful improvement over indirect or atlas-based targeting.

Direct Imaging of White-Matter Tracts Using dDTT

Twelve studies using deterministic tractography were included, 9 with clinical correlation. Findings are summarized in Table 4.
Studies using deterministic tractography to visualize the DRTT or CTCT are heterogeneous in their selection of ROIs, including, in various combinations, the cerebellar hemisphere, dentate nucleus, or SCP and the RN or thalamus, with or without inclusion of motor or premotor cortex as a third ROI. The cerebellar ROI is usually contralateral to the thalamus of interest, although ipsilateral seed points (corresponding to the nondecussating portion of the DRTT) have also been used [74]. This heterogeneity of ROIs as well as other dDTT parameters leads to difficulty in cross-study comparison.

Coenen et al. [75] demonstrated that effective Vim DBS contact locations were closely related to the DRTT, as defined using ROIs in the dentate nucleus and precentral gyrus (Fig. 5). Analysis was retrospective after standard indirect targeting and physiology-guided lead placement in 11 patients. A linear relationship between DBS efficacy and proximity to the center of the DRTT was found when elec- 

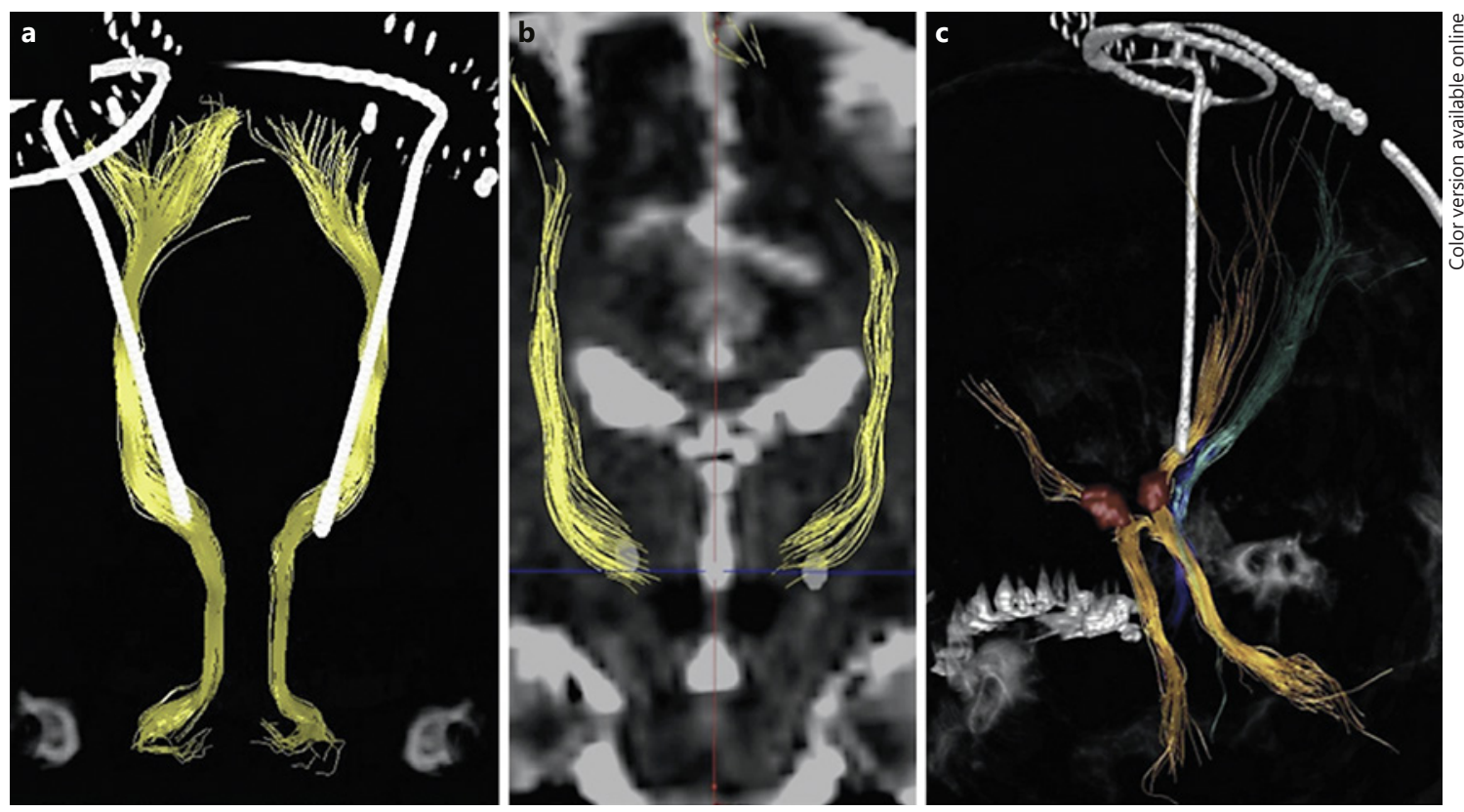

Fig. 5. Deterministic diffusion-tensor tractography. The dentato-rubro-thalamic tract is shown in relationship to implanted DBS electrodes (a), coronal MRI (b), and nearby structures such as the RN and medial lemniscus (c). Reproduced with permission from Coenen et al. [75].

trode locations and estimated VTAs were studied in standard Montreal Neurological Institute space. Importantly, this relationship was consistent at varying depths above and below the intercommissural line, thus covering the DRTT in the subthalamic area as well as within the thalamus. Schlaier et al. [76], using ROIs in the dentate, SCP, and $\mathrm{RN}$, also found effective contact location to be related to the DRTT, with more effective contacts located in, or lateral, posterior, and inferior to, the DRTT. Subsequent work by the same group [77] compared dDTT with pDTT in the same dataset, using ROIs for both the DRTT and CTCT and both 12- and 64-direction DTI, comparing all combinations with a standard indirect target. Both tracts were consistently found to be $\sim 2 \mathrm{~mm}$ anterior to indirect target, with the CTCT slightly posterior to the DRTT. Probabilistic methods were more sensitive in detecting the DRTT than dDTT but took considerably more processing time (12-33 h vs. 11-18 $\mathrm{min}$ ). This paper highlights the importance of ROI and parameter selection as well as the need for consistency across studies.

In a series of $10 \mathrm{ET}$ patients who underwent either thalamic DBS or thalamotomy using indirect targeting and awake physiology, Sasada et al. [78] retrospectively analyzed lesion and effective contact locations with respect to the DRTT as defined using both M1 and premotor target ROIs with SCP and thalamic seeds. Twelve of 14 effective contacts/lesions were found to be in the cerebello-thalamo-premotor tract. No information was provided as to intraoperative adjustments or differences between initial indirect targeting and final location in the DRTT.

Fenoy and Schiess [79] published the first prospective series using the DRTT as the initial target for intraoperative physiologic mapping and DBS placement. ROIs were selected as per Coenen et al. [75]. In comparison to the indirect target that would have been used in these patients, $\mathrm{X}-\mathrm{Y}$ coordinates were similar, but the tractography-derived target was $\sim 1 \mathrm{~mm}$ deeper $(\mathrm{Z}=-0.9)$. However, the active contacts were generally located above the intercommissural line, suggesting that thalamic stimulation might still have been the mechanism of efficacy. Unfortunately, no report was made of whether intraoperative adjustment of the planned trajectory was needed, nor whether such adjustments were more or less common compared to indirect targeting in the authors' previous experience. A follow-up study by the same authors [80] compared the 20 patients targeted using tractography with a previous, non-matched cohort of patients undergoing microelectrode recording based on a standard Vim target, noting similar tremor reduction but lower power use in the DRTT group compared to the Vim group. Again, efficiency of intraoperative mapping was not compared between the 2 groups. 
Table 5. Resting-state fMRI studies

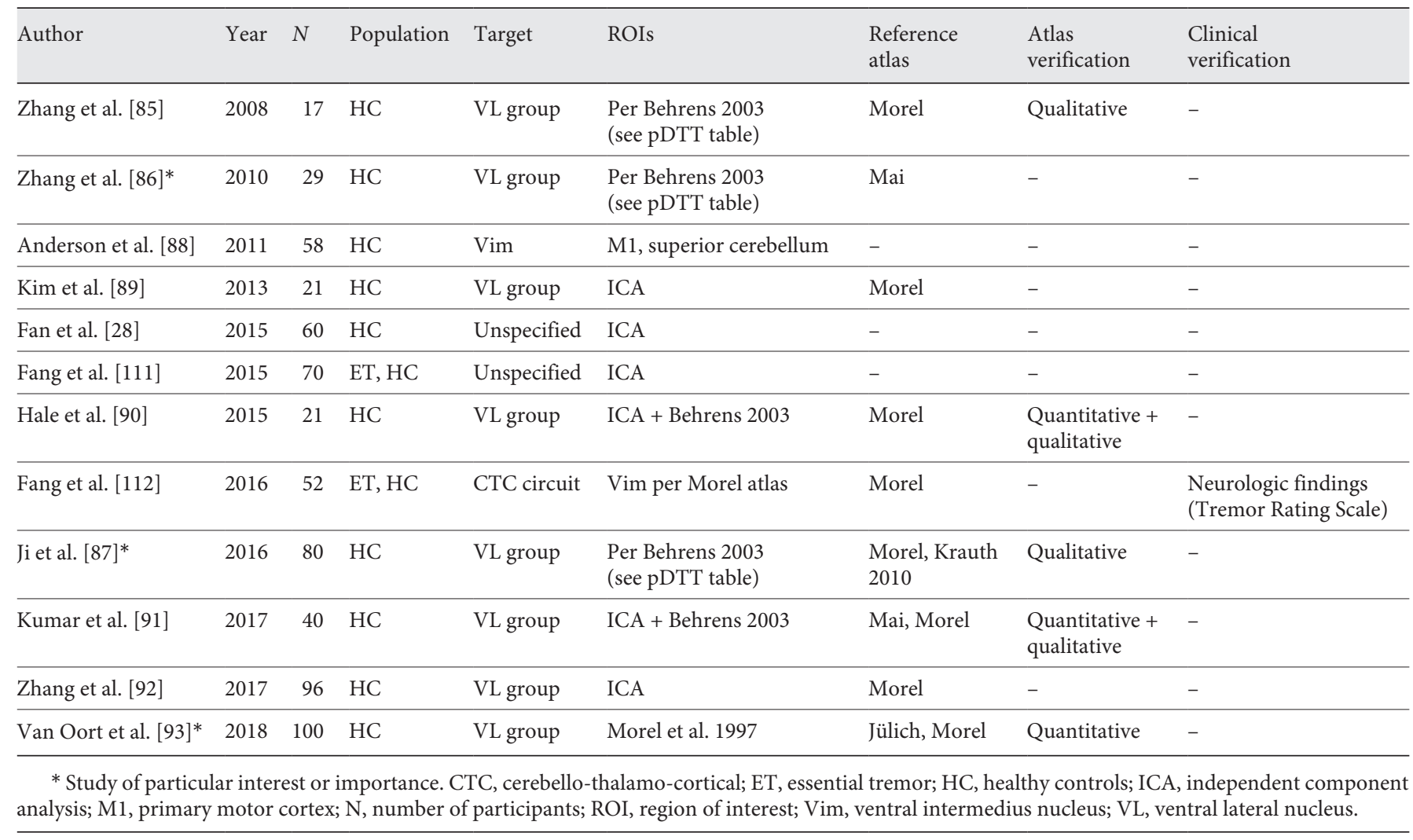

In $3 \mathrm{ET}$ patients who underwent awake thalamic DBS placement, Avecillas-Chasin et al. [81] combined whitematter tractography with gray-matter targeting, defining the Vim as the intersection of the DRTT with the thalamus. The locations of the clinically effective contacts were found to be significantly closer to the tractography-defined Vim coordinates than they were to the original indirect target. Chazen et al. [82] similarly targeted the intersection of the DRTT (generated from RN and cortical ROIs as per Yamada et al. [83]) with the thalamus as the target for magnetic resonance-guided focused ultrasound (MRgFUS) thalamotomy. In contrast, however, the tractography-based target was found to be within $1 \mathrm{~mm}$ of standard indirect coordinates.

Nowacki et al. [84] retrospectively evaluated 4 different ROI combinations to define the DRTT in 6 patients with ET who had undergone standard awake Vim DBS placement. Results were compared to the Morel atlas and were correlated with clinical outcome. ROIs were as follows: (1) ipsilateral dentate, ipsilateral SCP, and ipsilateral M1 (similar to Coenen et al. [75]); (2) contralateral dentate, contralateral SCP, and ipsilateral RN (similar to
Kwon et al. [69]); (3) ipsilateral dentate and ipsilateral PSA; and (4) ipsilateral dentate, ipsilateral PSA, and ipsilateral motor cortex. For each patient, diffusion-tensor parameters were adjusted until a biologically plausible tract was seen. Statistically significant differences among the 4 methods were noted in the lateral and anteroposterior coordinates of the DRTT within the subthalamic area. No consistent relationship was found between the location of the active contact (here, in the subthalamic area at approximately $Z=-2$ rather than the thalamus) and the DRTT by any of the 4 methods, each of which had significant variability with respect to active contact location. VTA calculations were not performed, raising the possibility that relationships between active contacts and tractography results might emerge when stimulation parameters are taken into account. The least amount of variability was seen when using ROIs in the dentate, the PSA, and ipsilateral motor cortex.

\section{Resting-State fMRI}

Twelve articles were included and are summarized in Table 5. 

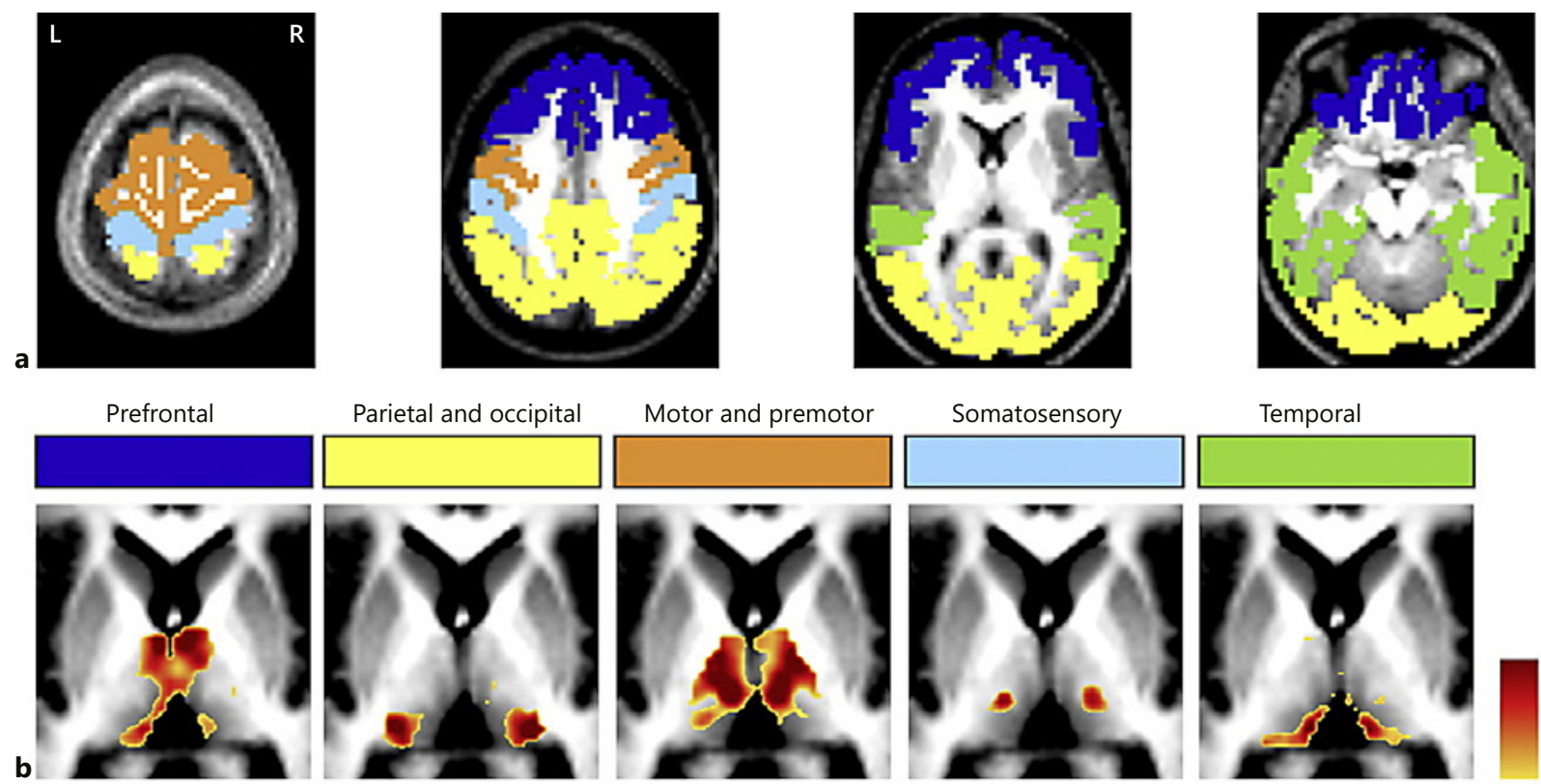

Somatosensory

Temporal
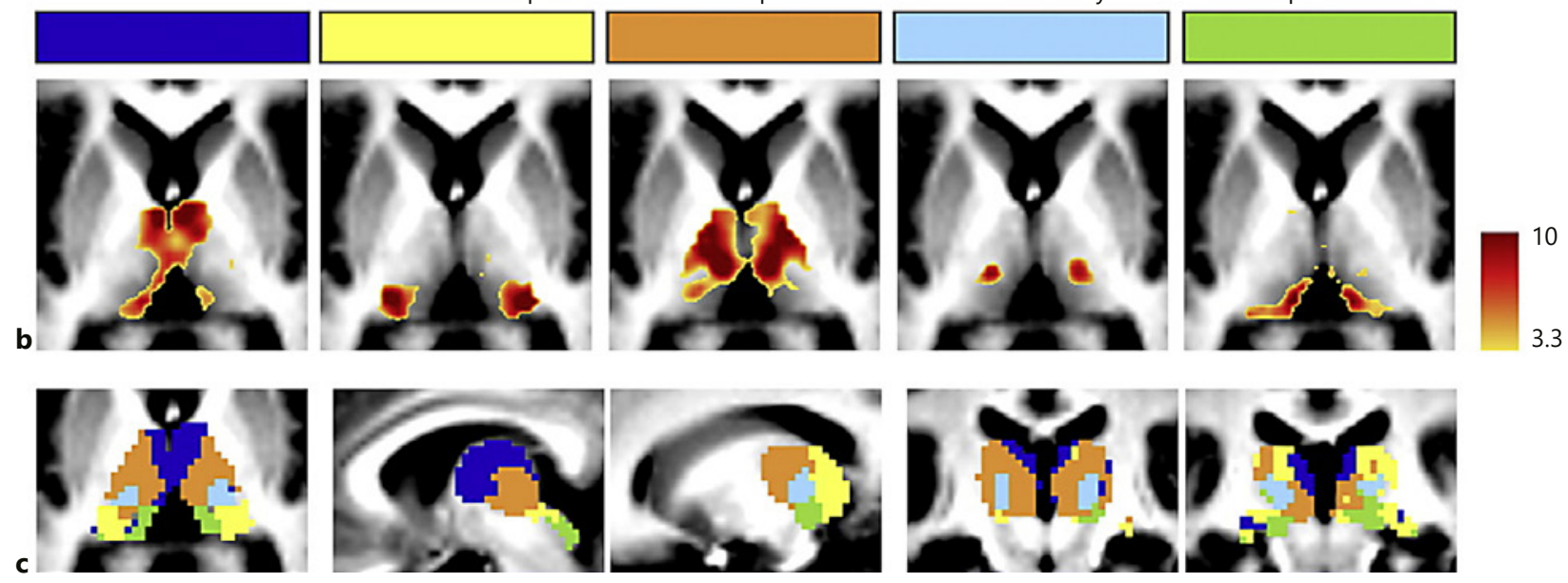

Fig. 6. Resting-state functional MRI. Time-dependent blood oxygen-level-dependent signal changes in predefined cortical areas (a) are correlated with those in the thalamus. Thalamic voxels showing high correlation with each cortical area are shown using heat maps (b, second row). Voxels are then assigned in a winner-take-all analysis and combined to form the subnuclear maps shown in (c). Reproduced with permission from Zhang et al. [85].

In 2008, Zhang et al. [85] provided the first major report of rs-fMRI parcellation of the thalamus. Functional correlation between each thalamic voxel and 5 predetermined cortical regions was explored, with cortical divisions outlined as in the tractography literature (i.e., per Behrens et al. [26]). Each thalamic voxel was found to be highly correlated with only 1 cortical region, suggesting distinct physiologic relevance. The resulting map of 5 thalamic regions, moreover, corresponded well to the expected nuclear group locations on the Morel atlas and known physiologic connections (Fig. 6). In particular, the voxels that were highly correlated with the primary motor and premotor cortices included the ventrolateral thalamus, as would be expected. Interestingly, bilateral thalamic correlation was seen with both unilateral and bilateral cortical ROIs, suggesting the presence of (possibly polysynaptic) interthalamic or transcallosal connections. In a follow-up study, the same group [86] demonstrated significant overlap between their rs-fMRI-derived segmentation and pDTT-derived segmentation. Although this analysis used pooled, rather than individual, subject data, the fact that structural and functional CDRs overlapped with each other more so than a histologic atlas mutually supports the 2 methods and is strong evidence for a structural - functional correlation.

A variation on the Zhang technique was used by Ji et al. [87], who analyzed the same 5 cortical ROIs in relationship to the thalamus in 9 temporally separate thalamocortical "states," combining the resulting maps to more accurately reflect functional correlation over time. This yielded improved correlation with the Morel atlas but required the pooling of data from a large number of healthy controls and extensive post-processing.

Greater spatial resolution within the motor thalamus was obtained by Anderson et al. [88] by correlating thalamic BOLD activity with both a primary motor cortex 
Table 6. Advanced indirect targeting studies

\begin{tabular}{lcclllll}
\hline Author & Year & $N$ & Population & Target & $\begin{array}{l}\text { Reference } \\
\text { atlas }\end{array}$ & $\begin{array}{l}\text { Atlas } \\
\text { verification }\end{array}$ & $\begin{array}{l}\text { Clinical } \\
\text { verification }\end{array}$ \\
\hline Lefranc et al. [94] & 2015 & 20 & ET, PD & Vim & S+W & Quantitative & SRS (retrospective) \\
\hline Sammartino et al. [5] & 2016 & 40 & $\begin{array}{l}\text { ET or tremor- } \\
\text { dominant PD; HC }\end{array}$ & Vim & $\begin{array}{l}\text { Indirect coordinates without } \\
\text { specific atlas reference }\end{array}$ & Quantitative & $\begin{array}{l}\text { EP, DBS, RF lesion } \\
(n=6) \text { retrospective) }\end{array}$ \\
\hline King et al. [95] & 2017 & 6 & ET, PD & Vim & S+W & Qualitative & EP (retrospective) \\
\hline Krishna et al. [96]* & 2019 & 10 & ET & Vim & - & - & FUS (prospective) \\
\hline
\end{tabular}

* Study of particular interest or importance. DBS, deep brain stimulation; EP, electrophysiology; ET, essential tremor; FUS, focused ultrasound thalamotomy; HC, healthy controls; N, number of participants; PD, Parkinson's disease; RF, radiofrequency; SRS, stereotactic radiosurgery; S+W, Schaltenbrand-Wahren; Vim, ventral intermedius nucleus.

ROI and a superior cerebellar motor ROI (as demonstrated by a finger-movement fMRI task). The thalamic voxel with highest functional connectivity to the 2 ROIs was within $3 \mathrm{~mm}$ of the expected location of the Vim. Results were pooled from 58 volunteers; in a simultaneous experiment, similar results were obtained from a single volunteer using approximately $3 \mathrm{~h}$ of fMRI imaging time.

Data from rs-fMRI can also be analyzed using ICA, which groups voxels based on the intrinsic similarity of their BOLD signal rather than their correlation with a prespecified cortical ROI. Although the number of independent components (IC) in a given structure must be pre-specified, this technique is otherwise relatively hypothesis free. Several groups have used ICA either alone [89] or in combination with ROI-based methods [90-92] to identify functional subunits of the thalamus and their correlation with cortical areas. Results have been mixed, with the boundaries of ICs generally showing only weak agreement with histological nuclei. It is unclear whether this lack of correlation between the boundaries of ICAderived thalamic units and histological nuclei represents error or variability in rs-fMRI technique or, alternatively, represents the fact that true functional divisions may not respect histologic boundaries. As described by van Oort et al. [93], Instantaneous Connectivity Parcellation, a variant of ICA using time signatures for parcellation, shows better correlation to structural outlines. Overall, as with much of $\mathrm{fMRI}$ research, clarifying the interpretation of BOLD signal as a marker of function will likely require prospective studies and clinical confirmation.

\section{Advanced Indirect Targeting}

We apply the term advanced indirect targeting to hybrid techniques that use indirect targeting (i.e., fixed numerical relationships) with reference to advanced-imag- ing features rather than to traditional landmarks such as the commissures and third ventricular wall. Studies in this group are summarized in Table 6 .

Lefranc et al. [94] retrospectively examined DTI in 20 patients who had undergone radiosurgical Vim thalamotomy and were selected for good response $(>80 \%$ tremor reduction). The prelemniscal radiations (Raprl), which are known to abut the inferior - posterior aspect of the Vim, were found to be reliably visible on color fractional anisotropy maps and consistently related to the lesion as well as to expected location based on the Schaltenbrand-Wahren atlas, suggesting that the Raprl may serve as an internal fiducial landmark in Vim localization. The observed location was also consistent on the Schaltenbrand-Wahren atlas; however, and no comparison or control group was presented to compare the additive value of Raprl visualization.

In 18 nonoperative ET patients and 6 awake surgical cases (3 ET, 3 PD; 2 DBS, 4 RF thalamotomy), Sammartino et al. [5] identified the pyramidal tract and medial lemniscus using $\mathrm{dDTT}$, then defined the tractographybased Vim (T-Vim) as a $4 \times 4 \times 5 \mathrm{~mm}$ ROI placed $3 \mathrm{~mm}$ medial to the pyramidal tract and $3 \mathrm{~mm}$ anterior to the medial lemniscus. The structural connectivity of the T-Vim ROI was subsequently investigated using pDTT, showing connection to the contralateral cerebellum in 19 of 26 hemispheres and to the ipsilateral M1 in 25 of 26 hemispheres; however, we consider this method advanced indirect targeting because the ROI is not defined by connectivity but by a fixed relationship to other structures. The T-Vim target was found to be $\sim 1 \mathrm{~mm}$ lateral and $\sim 2 \mathrm{~mm}$ anterior to the scaled indirect target by Guiot's coordinates, with mean Euclidean distance between T-Vim and surgically-defined Vim of $1.6 \mathrm{~mm}$. This correctly predicted the intraoperative adjustments made 
in 5 of the 6 surgical cases. A subsequent study [95] provided electrophysiologic support for this targeting method. The same hybrid targeting technique was then used prospectively in a series of 10 patients undergoing MRgFUS thalamotomy for ET [96], showing good correlation of the putative Vim ROI with the primary motor CDR as defined by Behrens et al. [26] as well as effective tremor suppression with minimal adverse effects.

\section{Discussion}

Does Direct Targeting of the Motor Thalamus and DRTT Represent a Clinical Advance over Traditional Indirect or Histological Atlas-Based Targeting?

Visualization and direct targeting of the tremor network on a patient-specific basis clearly have more conceptual appeal than the use of traditional indirect targeting or targeting based on histologic atlases with few subjects [9-12]. In theory, direct targeting should yield greater efficacy and energy efficiency while decreasing or even eliminating the need for intraoperative physiologic verification. Direct targeting based on structural MRI has been widely accepted for asleep DBS of the subthalamic nucleus and globus pallidus internus, and individualized tractography has been extensively studied in neuromodulation for depression [97].

In the case of thalamic neuromodulation for tremor, however, a clinical benefit for direct targeting has not yet been demonstrated except in case reports [98]. Several studies reviewed above present conflicting or difficult-tointerpret data on whether refinement of indirect coordinates based on advanced imaging would significantly alter electrode location, shorten intraoperative mapping, or result in additional clinical benefit [99]. Furthermore, several series in recent years have shown clinical success using indirect targeting without physiologic feedback for thalamic DBS [13], stereotactic radiosurgery [16], and MRgFUS [17], raising the practical question of whether direct targeting is necessary.

Several factors likely contribute to the efficacy of indirect targeting of the Vim. In contrast to traditional consensus coordinates for the subthalamic nucleus and globus pallidus internus, indirect Vim targeting usually includes scaling to the commissural distance and third ventricle width, after which residual individual thalamic variation may be small. Additionally, since several effective targets in the tremor network are clustered within a relatively small space, imperfect targeting may still result in adequate clinical benefit via stimulation of an alternate target. Separately, known access points to the DRTT, such as the PSA and cZI, are visible on conventional structural MRI, which may make tractography of the DRTT of little added value for centers that favor a subthalamic target.

Our own expectation is that direct targeting of the thalamic tremor network will yield an incremental, yet clinically meaningful, improvement in the therapeutic window of DBS for tremor and in the clinical outcomes of lesioning procedures. Even small improvements in accuracy likely confer advantages in power consumption and side effect management and, on a population basis, are likely to yield lower rates of lead revision and treatment failure. Advanced imaging also improves the ability to treat patients with abnormal thalamic or brainstem anatomy by localizing functional targets noninvasively. Perhaps more importantly, the direct imaging of target structures may allow more accurate characterization of lead location across patients and centers, leading to more systematic comparison of different targets or stimulation paradigms. Finally, research into novel targets is likely to be driven by advanced imaging techniques, with the imaging serving as both the source of physiologic hypotheses and the link from those hypotheses to surgical targeting. This approach applies not only to tremor and other movement disorders but also to the wide range of neuropsychiatric disorders that may be treatable with DBS or lesioning techniques [97]. As such, advanced targeting techniques allow us both to refine our use of current targets and to move toward the next stage in the evolution of DBS targeting.

What Have We Learned about the Tremor Network from Advanced Imaging of the Motor Thalamus and DRTT?

Thus far, advanced imaging has largely been used to improve accuracy for known functional or anatomic targets, not to advance understanding of the pathophysiology of tremor. The use of histologic atlases to guide thalamic parcellation and the choice of known DRTT waypoints as ROIs for tractography both necessarily introduce a degree of circularity to the conclusions that can be drawn. However, fMRI data have been helpful in validating the role of the motor nuclear group in motor activity, with functional connectivity that correlates with the known anatomic connections of the thalamus and motor cortex. In addition, tractography has lent critical support to the hypothesis that DRTT stimulation is the common mechanism of action for different subthalamic targets [75]. Advanced imaging has clarified, without yet answer- 
ing, the question of the optimal thalamic target, with some studies favoring primary motor-associated region (corresponding to Vim) and others favoring the premotor/SMA-associated region (corresponding to Vop).

Which of the Advanced Techniques Reviewed above Shows the Greatest Promise for Clinical Application?

The optimal targeting technique depends on the target chosen, and the optimal surgical target for tremor is not yet known. However, some conclusions can be drawn from the above review.

Structural MRI is the most conceptually straightforward approach to direct targeting of thalamic nuclei. At ultrahigh-field strength, SWI-based sequences have been shown to be capable of individualized parcellation, approaching the resolution of "in vivo histology" [19]. 7T imaging, however, is limited by availability and cost, the time and expertise required to produce high-quality images and to perform manual segmentation, local susceptibility artifacts from surrounding vessels [6], and the geometric distortion of high-field MRI systems, although such distortion may be correctable by coregistration [100]. At clinical field strengths, MRI sequences sensitive to myelin content can consistently delineate the ventrolateral nuclear group but have not yet shown resolution at the level of the Vim or Vop. Until clinical-strength imaging is further refined, multipatient $7 \mathrm{~T}$-derived atlases likely represent the most immediately practical bridge between research-and clinical-grade imaging. In combination with optimized clinical-strength imaging and accelerated, automated registration [59], such atlases have the potential to immediately enter into prospective trials in comparison to traditional atlases and other targeting techniques. With most 7T data having been generated on healthy control subjects, specific libraries of patients with ET and PD will need to be generated for greatest fidelity.

The clinical significance of thalamic segmentation by intrinsic diffusivity (i.e., DTI) is unclear, with a high degree of interindividual variation seen in these studies, weak clinical correlation, and spatial resolution generally inadequate for surgical targeting. To us, the assumption that individual nuclei will be identifiable solely based on anisotropic directionality seems flawed in a structure that contains many overlapping relay points and multiple inputs and outputs for each functional unit. Further evidence would be needed to validate this approach from both a conceptual and practical standpoint. The inherent spatial limitation and distortion of echo-planar imaging, upon which DTI is based, are another theoretical barrier to utility.
Deterministic tractography is in widespread use, is familiar to most surgeons, and has already been applied in clinical practice for neuromodulation of tremor. It is therefore well-positioned for inclusion in prospective trials. Since it is by definition limited to white-matter tracts, dDTT would be a targeting method of choice if the DRTT emerges as the most efficient target for control of tremor; however, the comparison between tract and nuclear stimulation will need to be made formally and prospectively.

Probabilistic tractography can be reliably applied in individual patients to separate the thalamus into CDRs. The premotor and primary motor CDRs appear to closely, but not perfectly, align with atlas-based definitions of the Vop and Vim, respectively - although this may, in fact, represent the limitations of atlas-based targeting. Whether pDTT-based targeting will be superior to traditional MRI or functional imaging is an empiric question; however, structural connectivity seems highly promising as a targeting technique once appropriate software and computing power are available for clinical use.

Resting-state $f M R I$ is a novel, in vivo window on normal and abnormal brain function and functional connectivity. Rs-fMRI studies have supported the motor function of the ventrolateral thalamic nuclei. Although theoretically attractive for targeting, rs-fMRI is unlikely to be practically useful in the near future for several technical reasons: most studies represent group-level analyses, and individual-level data can only be extracted with prolonged scan times and intensive data postprocessing; existing studies have utilized only healthy volunteers, limiting their application to pathologic states; and spatial resolution is generally limited to $2-$ $3 \mathrm{~mm}$ voxels, limiting its utility for surgical targeting. Thus, rs-fMRI is unlikely to be useful for individual refinement of known targets such as the Vim. However, its exploratory nature and the ability to investigate brain function in a relatively hypothesis-free manner may provide valuable information to guide the discovery of novel targets in movement disorders and other neuropsychiatric diseases.

Overall, multipatient 7T-derived atlases and dDTT of the DRTT appear best poised for clinical trials [101], with pDTT not far behind. The use of complementary grayand white-matter imaging may provide robustness to variations in technique.

\section{Future Directions}

Existing clinical data in surgical targeting for tremor suffer from heterogeneity in patient selection, surgical techniques, and outcome measurements. Although prospective studies comparing existing targets or targeting methods, as well as novel targets, would need careful 
methods to account for patient-related heterogeneity, it seems clear that the preliminary work is in place to conduct well-designed randomized clinical trials in tremor that address current targets and the targeting methods reviewed above. Primary endpoints should include tremor control (at least 1 year, but preferably 2 years after implant, both by blinded assessment and objective measurements), the rate of known stimulation-induced side effects such as dysarthria and imbalance, patient-reported quality-of-life measures, programming efficiency and power consumption. MRgFUS, which allows incisionless and reversible test lesioning, may facilitate comparison, albeit within the short timescale of a surgical session, and with the caveat that lesioning likely does not produce the same network effect as stimulation.

Specific goals of future prospective studies should include (1) systematic comparison of targets as defined by (a) structural imaging, (b) advanced 3-dimensional atlases, and (c) structural and functional connectivity; (2) systematic comparison of stimulation of the cerebello-thalamo-cortical circuit at its white-matter tract vs. thalamic nuclear (and possibly cortical) waypoints; (3) comparison of the efficacy of premotor (i.e., Vop) vs. primary motor (i.e., Vim) stimulation; (4) systematic comparison of probabilistic vs. deterministic tractography techniques for thalamic parcellation; and (5) exploration of novel targets and biomarkers of tremor subtypes based on advanced imaging. Although several imaging methods can be compared in individual subjects, target comparison, and clinical validation with DBS or lesioning will require careful matching or randomization techniques to account for different disease states, ages, type of tremor, and rate of progression. Study designs incorporating multiple electrode placement in individual patients would be reasonable given the context of clinical equipoise and known failure rates of single-target interventions over time.

Because advanced imaging depends highly on both image acquisition and post-processing, accurate comparison will require consistency in imaging parameters and acquisition, software choice and settings, and post-processing techniques, including coregistration. Prospective multi-institutional collaboration would greatly speed the acquisition and translation of useful clinical data.

\section{Conclusions}

Image-based thalamic parcellation has been the topic of extensive investigation over recent years, due to the proposed surgical benefits of more precise targeting, bet- ter outcomes, avoidance of physiologic mapping, and the simultaneous potential to develop biomarkers for degenerative and psychiatric disorders such as Alzheimer's disease, PD, schizophrenia, and multiple sclerosis [7]. We present a systematic review of the application of advanced imaging of the motor thalamus and identification of the DRTT following modified Preferred Reporting Items for Systematic Reviews and Meta-Analyses guidelines. These techniques are well-positioned for prospective study and comparison.

\section{Acknowledgments}

There are no acknowledgments to declare.

\section{Disclosure Statement}

The authors have no conflicts of interest to declare.

\section{Funding Sources}

No funding sources outside of our institution were utilized.

\section{Author Contributions}

N.G.: developed PubMed search strategy, screened publications for eligibility, reviewed publications and contributed to publication selection, authored draft manuscript, reviewed and formatted final manuscript. M.S.: reviewed publications, provided technical expertise regarding various advanced imaging techniques, contributed to manuscript development, reviewed final manuscript. N.P.: reviewed publications, revised manuscripts, reviewed final manuscript. W.S.K.: conceived of systematic review, screened publications for eligibility, finalized publication selection and interpretation, revised manuscripts, authored final manuscript.

\section{References} thalamic nuclei. In: Schaltenbrand G, Walker AE, editors. Stereotaxy of the human brain: anatomical, physiological, and clinical applications. New York: Thieme-Stratton; 1982. pp. $140-80$

2 Hirai T, Jones EG. A new parcellation of the cal staining. Brain Res Brain Res Rev. 1989 Jan-Mar;14(1):1-34.

3 Hirai T, Ohye C, Nagaseki Y, Matsumura M. Cytometric analysis of the thalamic ventralis intermedius nucleus in humans. J Neurophysiol. 1989 Mar;61(3):478-87.
1 Hassler R. Architectonic organization of the human thalamus on the basis of histochemi- 
4 Morel A, Magnin M, Jeanmonod D. Multiarchitectonic and stereotactic atlas of the human thalamus. J Comp Neurol. 1997 Nov; 387(4):588-630.

5 Sammartino F, Krishna V, King NK, Lozano AM, Schwartz ML, Huang Y, et al. Tractography-based ventral intermediate nucleus targeting: novel methodology and intraoperative validation. Mov Disord. 2016 Aug;31(8): 1217-25.

6 Najdenovska E, Tuleasca C, Jorge J, Maeder P, Marques JP, Roine T, et al. Comparison of MRI-based automated segmentation methods and functional neurosurgery targeting with direct visualization of the Ventro-intermediate thalamic nucleus at 7T. Sci Rep. 2019 Feb;9(1):1119.

7 Kelly PJ, Derome P, Guiot G. Thalamic spatial variability and the surgical results of lesions placed with neurophysiologic control. Surg Neurol. 1978 May;9(5):307-15.

8 Tourdias T, Saranathan M, Levesque IR, Su J, Rutt BK. Visualization of intra-thalamic nuclei with optimized white-matter-nulled MPRAGE at 7T. Neuroimage. 2014 Jan;84: 534-45.

9 Niemann K, Mennicken VR, Jeanmonod D, Morel A. The Morel stereotactic atlas of the human thalamus: atlas-to-MR registration of internally consistent canonical model. Neuroimage. 2000 Dec;12(6):601-16.

10 Atkinson JD, Collins DL, Bertrand G, Peters TM, Pike GB, Sadikot AF. Optimal location of thalamotomy lesions for tremor associated with Parkinson disease: a probabilistic analysis based on postoperative magnetic resonance imaging and an integrated digital atlas. J Neurosurg. 2002 May;96(5):854-66.

11 Krauth A, Blanc R, Poveda A, Jeanmonod D, Morel A, Székely G. A mean three-dimensional atlas of the human thalamus: generation from multiple histological data. Neuroimage. 2010 Feb;49(3):2053-62.

12 Sudhyadhom A, McGregor K, Okun MS, Foote KD, Trinastic J, Crosson B, et al. Delineation of motor and somatosensory thalamic subregions utilizing probabilistic diffusion tractography and electrophysiology. J Magn Reson Imaging. 2013 Mar;37(3):600-9.

13 Burchiel KJ, McCartney S, Lee A, Raslan AM. Accuracy of deep brain stimulation electrode placement using intraoperative computed tomography without microelectrode recording. J Neurosurg. 2013 Aug;119(2):301-6.

14 Chen T, Mirzadeh Z, Chapple KM, Lambert M, Evidente VG, Moguel-Cobos G, et al. Intraoperative test stimulation versus stereotactic accuracy as a surgical end point: a comparison of essential tremor outcomes after ventral intermediate nucleus deep brain stimulation. J Neurosurg. 2018 Aug;129(2): 290-8.

15 Brierley JB, Beck E. The significance in human stereotactic brain surgery of individual variation in the diencephalon and globus pallidus. J Neurol Neurosurg Psychiatry. 1959 Nov;22(4):287-98.
16 Kooshkabadi A, Lunsford LD, Tonetti D, Flickinger JC, Kondziolka D. Gamma Knife thalamotomy for tremor in the magnetic resonance imaging era. J Neurosurg. 2013 Apr; 118(4):713-8.

17 Elias WJ, Lipsman N, Ondo WG, Ghanouni P, Kim YG, Lee W, et al. A randomized trial of focused ultrasound thalamotomy for essential tremor. N Engl J Med. 2016 Aug; 375(8):730-9.

18 Abosch A, Yacoub E, Ugurbil K, Harel N. An assessment of current brain targets for deep brain stimulation surgery with susceptibilityweighted imaging at 7 tesla. Neurosurgery. 2010 Dec;67(6):1745-56.

19 Deistung A, Schäfer A, Schweser F, Biedermann U, Turner R, Reichenbach JR. Toward in vivo histology: a comparison of quantitative susceptibility mapping (QSM) with magnitude-, phase-, and R2*-imaging at ultrahigh magnetic field strength. Neuroimage. 2013 Jan;65:299-314.

20 Vassal F, Coste J, Derost P, Mendes V, Gabrillargues J, Nuti C, et al. Direct stereotactic targeting of the ventrointermediate nucleus of the thalamus based on anatomic 1.5-T MRI mapping with a white matter attenuated inversion recovery (WAIR) sequence. Brain Stimul. 2012 Oct;5(4):625-33.

21 Zerroug A, Gabrillargues J, Coll G, Vassal F, Jean B, Chabert E, et al. Personalized mapping of the deep brain with a white matter attenuated inversion recovery (WAIR) sequence at 1.5-tesla: experience based on a series of 156 patients. Neurochirurgie. 2016 Aug; 62(4):183-9.

22 Wiegell MR, Tuch DS, Larsson HB, Wedeen VJ. Automatic segmentation of thalamic nuclei from diffusion tensor magnetic resonance imaging. Neuroimage. 2003 Jun;19(2 Pt 1): 391-401.

23 Mang SC, Busza A, Reiterer S, Grodd W, Klose AU. Thalamus segmentation based on the local diffusion direction: a group study. Magn Reson Med. 2012 Jan;67(1):118-26.

24 Kumar V, Mang S, Grodd W. Direct diffusionbased parcellation of the human thalamus. Brain Struct Funct. 2015;220(3):1619-35.

25 Battistella G, Najdenovska E, Maeder P, Ghazaleh N, Daducci A, Thiran JP, et al. Robust thalamic nuclei segmentation method based on local diffusion magnetic resonance properties. Brain Struct Funct. 2017 Jul; 222(5):2203-16.

26 Behrens TE, Johansen-Berg H, Woolrich MW, Smith SM, Wheeler-Kingshott CA, Boulby PA, et al. Non-invasive mapping of connections between human thalamus and cortex using diffusion imaging. Nat Neurosci. 2003 Jul;6(7):750-7.

27 Akram H, Dayal V, Mahlknecht P, Georgiev D, Hyam J, Foltynie T, et al. Connectivity derived thalamic segmentation in deep brain stimulation for tremor. Neuroimage Clin. 2018 Jan;18:130-42.

28 Fan Y, Nickerson LD, Li H, Ma Y, Lyu B, Miao $\mathrm{X}$, et al. Functional connectivity-based parcel- lation of the thalamus: an unsupervised clustering method and ts Validity Investigation. Brain Connect. 2015 Dec;5(10):620-30.

29 O'Muircheartaigh J, Keller SS, Barker GJ, Richardson MP. White matter connectivity of the thalamus delineates the functional architecture of competing thalamocortical systems. Cereb Cortex. 2015 Nov;25(11):447789.

30 Hardy TL, Smith JR, Brynildson LR, Flanigan HF, Gray JG, Spurlock D. Magnetic resonance imaging and anatomic atlas mapping for thalamotomy. Stereotact Funct Neurosurg. 1992; 58(1-4):30-2.

31 Nowinski WL, Belov D, Thirunavuukarasuu A, Benabid AL. A probabilistic functional atlas of the VIM nucleus constructed from pre-, intra- and postoperative electrophysiological and neuroimaging data acquired during the surgical treatment of Parkinson's disease patients. Stereotact Funct Neurosurg. 2005;83(5-6):190-6.

32 Lorenzen P, Davis B, Joshi S. Unbiased atlas formation via large deformations metric mapping. Med Image Comput Comput Assist Interv. 2005;8(Pt 2):411-8.

33 Christensen GE, Johnson HJ, Vannier MW. Synthesizing average 3D anatomical shapes. Neuroimage. 2006 Aug;32(1):146-58.

34 Salanova V. Deep brain stimulation for epilepsy. Epilepsy Behav. 2018 Nov;88S:21-4.

35 Owen SL, Heath J, Kringelbach M, Green AL, Pereira EA, Jenkinson N, et al. Pre-operative DTI and probabilisitic tractography in four patients with deep brain stimulation for chronic pain. J Clin Neurosci. 2008 Jul;15(7):801-5.

36 Elias WJ, Zheng ZA, Domer P, Quigg M, Pouratian $\mathrm{N}$. Validation of connectivity-based thalamic segmentation with direct electrophysiologic recordings from human sensory thalamus. Neuroimage. 2012 Feb;59(3):2025-34.

37 Hunsche S, Sauner D, Runge MJ, Lenartz D, El Majdoub F, Treuer H, et al. Tractographyguided stimulation of somatosensory fibers for thalamic pain relief. Stereotact Funct Neurosurg. 2013;91(5):328-34.

38 Kim W, Chivukula S, Hauptman J, Pouratian N. Diffusion tensor imaging-based thalamic segmentation in deep brain stimulation for chronic pain conditions. Stereotact Funct Neurosurg. 2016;94(4):225-34.

39 Glaister J, Carass A, Stough JV, Calabresi PA, Prince JL. Thalamus parcellation using multimodal feature classification and thalamic nuclei priors. Proc SPIE Int Soc Opt Eng. 2016; 9784.

40 Klein JC, Rushworth MF, Behrens TE, Mackay CE, de Crespigny AJ, D’Arceuil H, et al. Topography of connections between human prefrontal cortex and mediodorsal thalamus studied with diffusion tractography. Neuroimage. 2010 Jun;51(2):555-64.

41 Jang SH, Yeo SS. Thalamocortical connections between the mediodorsal nucleus of the thalamus and prefrontal cortex in the human brain: a diffusion tensor tractographic study. Yonsei Med J. 2014 May;55(3):709-14. 
42 Magon S, Chakravarty MM, Amann M, Weier K, Naegelin Y, Andelova M, et al. Labelfusion-segmentation and deformation-based shape analysis of deep gray matter in multiple sclerosis: the impact of thalamic subnuclei on disability. Hum Brain Mapp. 2014 Aug;35(8): 4193-203.

43 Bisecco A, Rocca MA, Pagani E, Mancini L, Enzinger C, Gallo A, et al.; MAGNIMS Network. Connectivity-based parcellation of the thalamus in multiple sclerosis and its implications for cognitive impairment: A multicenter study. Hum Brain Mapp. 2015 Jul;36(7): 2809-25.

44 Coenen VA, Schlaepfer TE, Allert N, Mädler B. Diffusion tensor imaging and neuromodulation: DTI as key technology for deep brain stimulation. Int Rev Neurobiol. 2012;107: 207-34.

45 Zarei M, Patenaude B, Damoiseaux J, Morgese C, Smith S, Matthews PM, et al. Combining shape and connectivity analysis: an MRI study of thalamic degeneration in Alzheimer's disease. Neuroimage. 2010 Jan;49(1):1-8.

46 Sweet JA, Walter BL, Gunalan K, Chaturvedi A, McIntyre CC, Miller JP. Fiber tractography of the axonal pathways linking the basal ganglia and cerebellum in Parkinson disease: implications for targeting in deep brain stimulation. J Neurosurg. 2014 Apr;120(4):98896.

47 Henderson JM. "Connectomic surgery": diffusion tensor imaging (DTI) tractography as a targeting modality for surgical modulation of neural networks. Front Integr Nuerosci. 2012 Apr;6:15.

48 Moher D, Shamseer L, Clarke M, Ghersi D, Liberati A, Petticrew M, et al.; PRISMA-P Group. Preferred reporting items for systematic review and meta-analysis protocols (PRISMA-P) 2015 statement. Syst Rev. 2015 Jan;4(1):1.

49 Ramirez-Zamora A, Smith H, Kumar V, Prusik J, Phookan S, Pilitsis JG. Evolving concepts in posterior subthalamic area deep brain stimulation for treatment of tremor: surgical neuroanatomy and practical considerations. Stereotact Funct Neurosurg. 2016;94(5):28397.

50 Kawashima Y, Chen HJ, Takahashi A, Hirato M, Ohye C. Application of magnetic resonance imaging in functional stereotactic thalamotomy for the evaluation of individual variations of the thalamus. Stereotact Funct Neurosurg. 1992;58(1-4):33-8.

51 Magnotta VA, Gold S, Andreasen NC, Ehrhardt JC, Yuh WT. Visualization of subthalamic nuclei with cortex attenuated inversion recovery MR imaging. Neuroimage. 2000 Apr;11(4):341-6.

52 Gringel T, Schulz-Schaeffer W, Elolf E, Frölich A, Dechent P, Helms G. Optimized highresolution mapping of magnetization transfer (MT) at 3 Tesla for direct visualization of substructures of the human thalamus in clinically feasible measurement time. J Magn Reson Imaging. 2009 Jun;29(6):1285-92.
53 Sudhyadhom A, Haq IU, Foote KD, Okun MS, Bova FJ. A high resolution and high contrast MRI for differentiation of subcortical structures for DBS targeting: the Fast Gray Matter Acquisition T1 Inversion Recovery (FGATIR). Neuroimage. 2009 Aug;47 Suppl 2:T44-52.

54 Bender B, Mänz C, Korn A, Nägele T, Klose $\mathrm{U}$. Optimized 3D magnetization-prepared rapid acquisition of gradient echo: identification of thalamus substructures at 3T. AJNR Am J Neuroradiol. 2011 Dec;32(11):2110-5.

55 Patil AA, Falloon T, Hahn F, Cheng J, Wang S. Direct identification of ventrointermediate nucleus of the thalamus on magnetic resonance and computed tomography images. Surg Neurol. 1999 Jun;51(6):674-8.

56 Spiegelmann R, Nissim O, Daniels D, Ocherashvilli A, Mardor Y. Stereotactic targeting of the ventrointermediate nucleus of the thalamus by direct visualization with high-field MRI. Stereotact Funct Neurosurg. 2006; 84(1):19-23.

57 Young GS, Feng F, Shen H, Chen NK. Susceptibility-enhanced 3-Tesla T1-weighted spoiled gradient echo of the midbrain nuclei for guidance of deep brain stimulation implantation. Neurosurgery. 2009 Oct;65(4): 809-15.

58 Su J, Federau C, Tourdias T, Saranathan M, Halpern C, Henderson J, et al. White-matternulled MP-RAGE predicts clinical outcome of focused ultrasound thalamic ablation for essential tremor. Proceeding of the 24th annual meeting of International Society of Magnetic Resonance in Medicine, Singapore, 2016. p. 0554.

$59 \mathrm{Su} \mathrm{JH}$, Thomas FT, Kasoff WS, Tourdias T, Choi EY, Rutt BK, et al. Thalamus Optimized Multi Atlas Segmentation (THOMAS): fast, fully automated segmentation of thalamic nuclei from structural MRI. Neuroimage. 2019 Jul;194:272-82.

60 Guiot G, Hardy J, Albe-Fessard D. [Precise delimitation of the subcortical structures and identification of thalamic nuclei in man by stereotactic electrophysiology]. Neurochirurgia (Stuttg). 1962 Apr;5:1-18.

61 Yovel Y, Assaf Y. Virtual definition of neuronal tissue by cluster analysis of multi-parametric imaging (virtual-dot-com imaging). Neuroimage. 2007 Mar;35(1):58-69.

62 Deoni SC, Rutt BK, Parrent AG, Peters TM. Segmentation of thalamic nuclei using a modified k-means clustering algorithm and highresolution quantitative magnetic resonance imaging at 1.5 T. Neuroimage. $2007 \mathrm{Jan} ; 34(1)$ : $117-26$.

63 Unrath A, Klose U, Grodd W, Ludolph AC, Kassubek J. Directional colour encoding of the human thalamus by diffusion tensor imaging. Neurosci Lett. 2008 Apr;434(3):322-7.

64 Sedrak M, Gorgulho A, Frew A, Behnke E, DeSalles A, Pouratian N. Diffusion tensor imaging and colored fractional anisotropy mapping of the ventralis intermedius nucleus of the thalamus. Neurosurgery. 2011 Nov;69(5):1124-9.
65 Jakab A, Werner B, Piccirelli M, Kovács K, Martin E, Thornton JS, et al. Feasibility of diffusion tractography for the reconstruction of intra-thalamic and cerebello-thalamic targets for functional neurosurgery: a multi-vendor pilot study in four subjects. Front Neuroanat. 2016 Jul; 10:76.

66 Lambert C, Simon H, Colman J, Barrick TR. Defining thalamic nuclei and topographic connectivity gradients in vivo. Neuroimage. 2017 Sep;158:466-79.

67 Broser P, Vargha-Khadem F, Clark CA. Robust subdivision of the thalamus in children based on probability distribution functions calculated from probabilistic tractography. Neuroimage. 2011 Jul;57(2):403-15.

68 Johansen-Berg H, Behrens TE, Sillery E, Ciccarelli O, Thompson AJ, Smith SM, et al. Functional-anatomical validation and individual variation of diffusion tractographybased segmentation of the human thalamus. Cereb Cortex. 2005 Jan;15(1):31-9.

69 Kwon HG, Hong JH, Hong CP, Lee DH, Ahn $\mathrm{SH}$, Jang SH. Dentatorubrothalamic tract in human brain: diffusion tensor tractography study. Neuroradiology. 2011 Oct;53(10):78791.

70 O’Muircheartaigh J, Vollmar C, Traynor C, Barker GJ, Kumari V, Symms MR, et al. Clustering probabilistic tractograms using independent component analysis applied to the thalamus. Neuroimage. 2011 Feb;54(3):2020-32.

71 Pouratian N, Zheng Z, Bari AA, Behnke E, Elias WJ, Desalles AA. Multi-institutional evaluation of deep brain stimulation targeting using probabilistic connectivity-based thalamic segmentation. J Neurosurg. 2011 Nov; 115(5):995-1004.

72 Middlebrooks EH, Tuna IS, Almeida L, Grewal SS, Wong J, Heckman MG, et al. Structural connectivity-based segmentation of the thalamus and prediction of tremor improvement following thalamic deep brain stimulation of the ventral intermediate nucleus. Neuroimage Clin. 2018;20:1266-73.

73 Klein JC, Barbe MT, Seifried C, Baudrexel S, Runge M, Maarouf M, et al. The tremor network targeted by successful VIM deep brain stimulation in humans. Neurology. 2012 Mar; 78(11):787-95.

74 Meola A, Comert A, Yeh FC, Sivakanthan S, Fernandez-Miranda JC. The nondecussating pathway of the dentatorubrothalamic tract in humans: human connectome-based tractographic study and microdissection validation. J Neurosurg. 2016 May;124(5):1406-12.

75 Coenen VA, Allert N, Paus S, Kronenbürger M, Urbach H, Mädler B. Modulation of the cerebello-thalamo-cortical network in thalamic deep brain stimulation for tremor: a diffusion tensor imaging study. Neurosurgery. 2014 Dec;75(6):657-69.

76 Schlaier J, Anthofer J, Steib K, Fellner C, Rothenfusser E, Brawanski A, et al. Deep brain stimulation for essential tremor: targeting the dentato-rubro-thalamic tract? Neuromodulation. 2015 Feb;18(2):105-12. 
77 Schlaier JR, Beer AL, Faltermeier R, Fellner C, Steib K, Lange M, et al. Probabilistic vs. deterministic fiber tracking and the influence of different seed regions to delineate cerebellarthalamic fibers in deep brain stimulation. Eur J Neurosci. 2017 Jun;45(12):1623-33.

78 Sasada S, Agari T, Sasaki T, Kondo A, Shinko A, Wakamori T, et al. Efficacy of fiber tractography in the stereotactic surgery of the thalamus for patients with essential tremor. Neurol Med Chir (Tokyo). 2017 Aug;57(8):392-401.

79 Fenoy AJ, Schiess MC. Deep brain stimulation of the dentato-rubro-thalamic tract: outcomes of direct targeting for tremor. Neuromodulation. 2017 Jul;20(5):429-36.

80 Fenoy AJ, Schiess MC. Comparison of tractography-assisted to atlas-based targeting for deep brain stimulation in essential tremor. Mov Disord. 2018 Dec;33(12):1895-901.

81 Avecillas-Chasin JM, Alonso-Frech F, Parras O, Del Prado N, Barcia JA. Assessment of a method to determine deep brain stimulation targets using deterministic tractography in a navigation system. Neurosurg Rev. 2015 Oct;38(4):739-50.

82 Chazen JL, Sarva H, Stieg PE, Min RJ, Ballon DJ, Pryor KO, et al. Clinical improvement associated with targeted interruption of the cerebellothalamic tract following MR-guided focused ultrasound for essential tremor. J Neurosurg. 2018 Aug;129(2):315-23.

83 Yamada K, Akazawa K, Yuen S, Goto M, Matsushima $\mathrm{S}$, Takahata A, et al. MR imaging of ventral thalamic nuclei. AJNR Am J Neuroradiol. 2010 Apr;31(4):732-5.

84 Nowacki A, Schlaier J, Debove I, Pollo C. Validation of diffusion tensor imaging tractography to visualize the dentatorubrothalamic tract for surgical planning. J Neurosurg. 2018 Mar;130(1):99-108.

85 Zhang D, Snyder AZ, Fox MD, Sansbury MW, Shimony JS, Raichle ME. Intrinsic functional relations between human cerebral cortex and thalamus. J Neurophysiol. 2008 Oct; 100(4):1740-8

86 Zhang D, Snyder AZ, Shimony JS, Fox MD, Raichle ME. Noninvasive functional and structural connectivity mapping of the human thalamocortical system. Cereb Cortex. 2010 May;20(5):1187-94.

87 Ji B, Li Z, Li K, Li L, Langley J, Shen H, et al. Dynamic thalamus parcellation from restingstate fMRI data. Hum Brain Mapp. 2016 Mar; 37(3):954-67.

88 Anderson JS, Dhatt HS, Ferguson MA, LopezLarson M, Schrock LE, House PA, et al. Functional connectivity targeting for deep brain stimulation in essential tremor. AJNR Am J Neuroradiol. 2011 Nov-Dec;32(10):1963-8.

89 Kim DJ, Park B, Park HJ. Functional connectivity-based identification of subdivisions of the basal ganglia and thalamus using multilevel independent component analysis of resting state fMRI. Hum Brain Mapp. 2013 Jun; 34(6):1371-85
90 Hale JR, Mayhew SD, Mullinger KJ, Wilson RS, Arvanitis TN, Francis ST, et al. Comparison of functional thalamic segmentation from seed-based analysis and ICA. Neuroimage. 2015 Jul;114:448-65.

91 Kumar VJ, van Oort E, Scheffler K, Beckmann CF, Grodd W. Functional anatomy of the human thalamus at rest. Neuroimage. 2017 Feb;147:678-91.

92 Zhang S, Li CR. Functional connectivity parcellation of the human thalamus by independent component analysis. Brain Connect. 2017 Nov;7(9):602-16.

93 van Oort ES, Mennes M, Navarro Schröder T, Kumar VJ, Zaragoza Jimenez NI, Grodd $\mathrm{W}$, et al. Functional parcellation using time courses of instantaneous connectivity. Neuroimage. 2018 Apr;170:31-40.

94 Lefranc M, Carron R, Regis J. Prelemniscal radiations: a new reliable landmark of the thalamic nucleus ventralis intermedius location. Stereotact Funct Neurosurg. 2015; 93(6):400-6.

95 King NK, Krishna V, Basha D, Elias G, Sammartino F, Hodaie M, et al. Microelectrode recording findings within the tractographydefined ventral intermediate nucleus. J Neurosurg. 2017 May;126(5):1669-75.

96 Krishna V, Sammartino F, Agrawal P, Changizi BK, Bourekas E, Knopp MV, et al. Prospective tractography-based targeting for improved safety of focused ultrasound thalamotomy. Neurosurgery. 2019 Jan;84(1): $160-8$.

97 Riva-Posse P, Choi KS, Holtzheimer PE, McIntyre CC, Gross RE, Chaturvedi A, et al. Defining critical white matter pathways mediating successful subcallosal cingulate deep brain stimulation for treatment-resistant depression. Biol Psychiatry. 2014 Dec;76(12): 963-9.

98 O'Halloran RL, Chartrain AG, Rasouli JJ, Ramdhani RA, Kopell BH. Case study of image-guided deep brain stimulation: magnetic resonance imaging-based white matter tractography shows differences in responders and nonresponders. World Neurosurg. 2016 Dec;96:613.e9-16.

99 Anthofer J, Steib K, Fellner C, Lange M, Brawanski A, Schlaier J. The variability of atlas-based targets in relation to surrounding major fibre tracts in thalamic deep brain stimulation. Acta Neurochir (Wien). 2014 Aug;156(8):1497-504.

100 Duchin Y, Abosch A, Yacoub E, Sapiro G, Harel N. Feasibility of using ultra-high field (7 T) MRI for clinical surgical targeting. PLoS One. 2012;7(5):e37328.

101 Sajonz BE, Amtage F, Reinacher PC, Jenkner C, Piroth T, Kätzler J, et al. Deep brain stimulation for tremor tractographic versus traditional (DISTINCT): study protocol of a randomized controlled feasibility trial. JMIR Res Protoc. 2016 Dec;5(4):e244.
102 Ziyan U, Tuch D, Westin CF. Segmentation of thalamic nuclei from DTI using spectral clustering. Med Image Comput Comput Assist Interv. 2006;9(Pt 2):807-14.

103 Jonasson L, Hagmann P, Pollo C, Bresson X, Richero Wilson C, Meuli R, et al. A level set method for segmentation of the thalamus and its nuclei in DT-MRI. Signal Processing. 2007;87(2):309-21.

104 Ye C, Bogovic JA, Ying SH, Prince JL. Parcellation of the thalamus using diffusion tensor images and a multi-object geometric deformable model. Proc SPIE Int Soc Opt Eng. 2013;8669.

105 Hyam JA, Owen SL, Kringelbach ML, Jenkinson N, Stein JF, Green AL, et al. Contrasting connectivity of the ventralis intermedius and ventralis oralis posterior nuclei of the motor thalamus demonstrated by probabilistic tractography. Neurosurgery. 2012 Jan; 70(1):162-9.

106 Jakab A, Blanc R, Berényi EL, Székely G Generation of individualized thalamus target maps by using statistical shape models and thalamocortical tractography. AJNR Am J Neuroradiol. 2012 Dec;33(11):2110-6.

107 Kincses ZT, Szabó N, Valálik I, Kopniczky Z, Dézsi L, Klivényi $P$, et al. Target identification for stereotactic thalamotomy using diffusion tractography. PLoS One. 2012;7(1): e29969.

108 Middlebrooks EH, Holanda VM, Tuna IS, Deshpande HD, Bredel M, Almeida L, et al. A method for pre-operative single-subject thalamic segmentation based on probabilistic tractography for essential tremor deep brain stimulation. Neuroradiology. 2018 Mar;60(3):303-9.

109 Tsolaki E, Downes A, Speier W, Elias WJ, Pouratian N. The potential value of probabilistic tractography-based for MR-guided focused ultrasound thalamotomy for essential tremor. Neuroimage Clin. 2017 Dec; 17: 1019-27.

110 King NK, Krishna V, Sammartino F, Bari A Reddy GD, Hodaie M, et al. Anatomic targeting of the optimal location for thalamic deep brain stimulation in patients with essential tremor. World Neurosurg. 2017 Nov; 107:168-74.

111 Fang W, Chen $\mathrm{H}$, Wang H, Zhang H, Liu M, Puneet $\mathrm{M}$, et al. Multiple resting-state networks are associated with tremors and cognitive features in essential tremor. Mov Disord. 2015 Dec;30(14):1926-36.

112 Fang W, Chen $\mathrm{H}$, Wang $\mathrm{H}$, Zhang $\mathrm{H}$, Puneet $\mathrm{M}$, Liu M, et al. Essential tremor is associated with disruption of functional connectivity in the ventral intermediate Nucleus-Motor Cortex-Cerebellum circuit. Hum Brain Mapp. 2016 Jan;37(1):165-78. 